Article

\title{
Analysis of the Constituents in "Zhu She Yong Xue Shuan Tong" by Ultra High Performance Liquid Chromatography with Quadrupole Time-of-Flight Mass Spectrometry Combined with Preparative High Performance Liquid Chromatography
}

\author{
Lin-Lin Wang 1,2, Li-Feng Han 1,2, He-Shui Yu ${ }^{1}$, Mang-Mang Sang ${ }^{1,2}$, Er-Wei Liu 1,2, \\ Yi Zhang ${ }^{1,2}$, Shi-Ming Fang ${ }^{1,2}$, Tao Wang ${ }^{1,2}$ and Xiu-Mei Gao ${ }^{1, *}$ \\ Received: 5 September 2015 ; Accepted: 6 November 2015 ; Published: 18 November 2015 \\ Academic Editor: Derek J. McPhee \\ 1 Tianjin State Key Laboratory of Modern Chinese Medicine, Tianjin University of Traditional Chinese \\ Medicine, 312 Anshanxi Road, Nankai District, Tianjin 300193, China; lynnwlin@yeah.net (L.-L.W.); \\ hanlifeng_1@sohu.com (L.-F.H.); hs_yu08@163.com (H.-S.Y.); 1174662177sang@sina.com (M.-M.S.); \\ liuwei628@hotmail.com (E.-W.L.); zhwwxzh@263.net (Y.Z.); fang_shiming@163.com (S.-M.F.); \\ wangt@263.net (T.W.) \\ 2 Tianjin Key Laboratory of TCM Chemistry and Analysis, Tianjin University of Traditional Chinese Medicine, \\ 312 Anshanxi Road, Nankai District, Tianjin 300193, China \\ * Correspondence: gaoxiumei@tjutcm.edu.cn; Tel.: +86-22-5959-6163; Fax: +86-22-5959-6163
}

\begin{abstract}
Zhu She Yong Xue Shuan Tong" lyophilized powder (ZSYXST), consists of a series of saponins extracted from Panax notoginseng, which has been widely used in China for the treatment of strokes. In this study, an ultra-high performance liquid chromatography with quadrupole time-of-flight mass spectrometry (UHPLC-Q-TOF/MS) combined with preparative high performance liquid chromatography (PHPLC) method was developed to rapidly identify both major and minor saponins in ZSYXST. Some high content components were removed through PHPLC in order to increase the sensitivity of the trace saponins. Then, specific characteristic fragment ions in both positive and negative mode were utilized to determine the types of aglycone, saccharide, as well as the saccharide chain linkages. As a result, 94 saponins, including 20 pairs of isomers and ten new compounds, which could represent higher than $98 \%$ components in ZSYXST, were identified or tentatively identified in commercial ZSYXST samples.
\end{abstract}

Keywords: ZSYXST; saponins; UHPLC-Q-TOF/MS

\section{Introduction}

"Zhu She Yong Xue Shuan Tong" lyophilized powder, containing saponins of Panax notoginseng, is commonly used for treating strokes in the clinic. It can dilate blood vessels, promote blood circulation [1], and prevent thrombosis [2-4]. It is also reported to have a therapeutic effect on diabetes [5,6]. Its efficacy has been confirmed and is widely accepted in clinical application. The average annual sales of ZSYXST in China are about a hundred million dollars. Although it is widely used in China, its chemical constituents, especially the minor compounds, are not understood very well. Recently, there was a chemical analysis of ZSYXST by an LC/MS method, however, only 30 compounds were identified because the researchers only used a normal LC/MS method [7]. Thus, the aim of this study is to establish a comprehensive analytical method to profile the constituents of ZSYXST as much as possible. 
Ultra-high performance liquid chromatography (UHPLC) is characterized by the advantages of high resolution, good sensitivity, high speed of analysis and high peak capacity [8]. Quadrupole time of flight mass spectrometry (Q-TOF/MS) has already been widely used for structural characterization of unknown saponins [9-11]. Thus, combining UHPLC with Q-TOF/MS (UHPLC-Q-TOF/MS) could be an effective method to identify the chemical constituents in ZSYXST. There are four major saponins (notoginsenoside $\mathrm{R}_{1}$, ginsenosides $\mathrm{Rg}_{1}$, $\mathrm{Re}$, and $\mathrm{Rb}_{1}$ ) which together represent more than $85 \%$ in ZSYXST, and these four major saponins could significantly decrease the sensitivity of minor saponins in the LC-MS fingerprint. Preparative high performance liquid chromatography (PHPLC) is a separation and purification technology with the advantages of high separation efficiency, sensitive detection and automated collection of fractions. This study utilized PHPLC to remove the major ingredients from ZSYXST samples in order to decrease the influence of the major saponins on the MS detection of the minor saponins.

In this paper, the structural characteristics of saponins from ZSYXST were investigated and illuminated using UHPLC-Q-TOF/MS and a target MS/MS data acquisition strategy. The full MS scan provided protonated or deprotonated molecules in their intact form, while the target MS/MS scan provided fragment information. The fragmentation patterns of the reference saponins were investigated first, and the types of aglycone, sequences and linkage positions of saccharide chains could be deduced accurately according to some diagnostic fragments pathways in both positive and negative modes. Finally, with the help of fragmentation pathway rules and finding compounds by the molecule feature in the Agilent Mass Hunter Workstation Software (Version B.02.00), 94 saponins, including 20 pairs of isomers, which could represent more than $98 \%$ of the components were identified or tentatively identified in commercial ZSYXST samples. Based on the literature and the SciFinder database, compounds 1, 2, 18, 20, 21, 22, 50, 51, 59, 90 were speculated to be new saponins in ZSYXST.

\section{Results and Discussion}

\subsection{Optimization of MS Conditions}

In order to obtain better MS response, cone voltage and CE were optimized. According to our research and literature data [12-15], the cone voltage was set to $120 \mathrm{~V}$ and $175 \mathrm{~V}$ in positive and negative mode, respectively. CE was dynamically adjusted from 45 to $70 \mathrm{~V}$ according to the $\mathrm{m} / \mathrm{z}$ of precursor ions in the negative MS/MS mode, such as $45 \mathrm{~V}$ for $\mathrm{m} / \mathrm{z} 650-750,50 \mathrm{~V}$ for $\mathrm{m} / z$ 750-850, $55 \mathrm{~V}$ for $m / z$ 850-950, $60 \mathrm{~V}$ for $m / z$ 950-1050, 65 or $70 \mathrm{~V}$ for $m / z>1050$.

\subsection{MS Cleavage Rules of Saponins in ZSYXST}

According to cleavage pathway of reference compounds and literatures, some diagnostic rules for the identification of compounds in ZSYXST can be summarized.

\subsubsection{Differentiation and Classification of Diverse Saponins in ZSYXST}

There were three main types of saponins in ZSYXST (Figure 1), namely protopanaxadiol (PPD), protopanaxatriol (PPT), and ocotillol (OCO) types [16,17]. According to the literature [12,15], there are some fragmentation rules for the sugar chains of saponins in $P$. notoginseng. The sugar moieties of PPD type are usually attached at the C-3 or C-3, 20 positions, while PPT sugars are attached at C-6 or C-6, C-20. OCO type usually form glycosides at the C-6 position when there as a five membered epoxy ring at C-20 [18-20]. According to [12,21] and our results, some characteristic ions could be used for deducing aglycone types, for example, PPD-type could be identified by diagnostic fragment ions at $m / z 443,425$ and 407, PPT-type by $m / z 441,423$ and 405, and OCO-type by $m / z 457,439$ and 421 (Figure 1). 


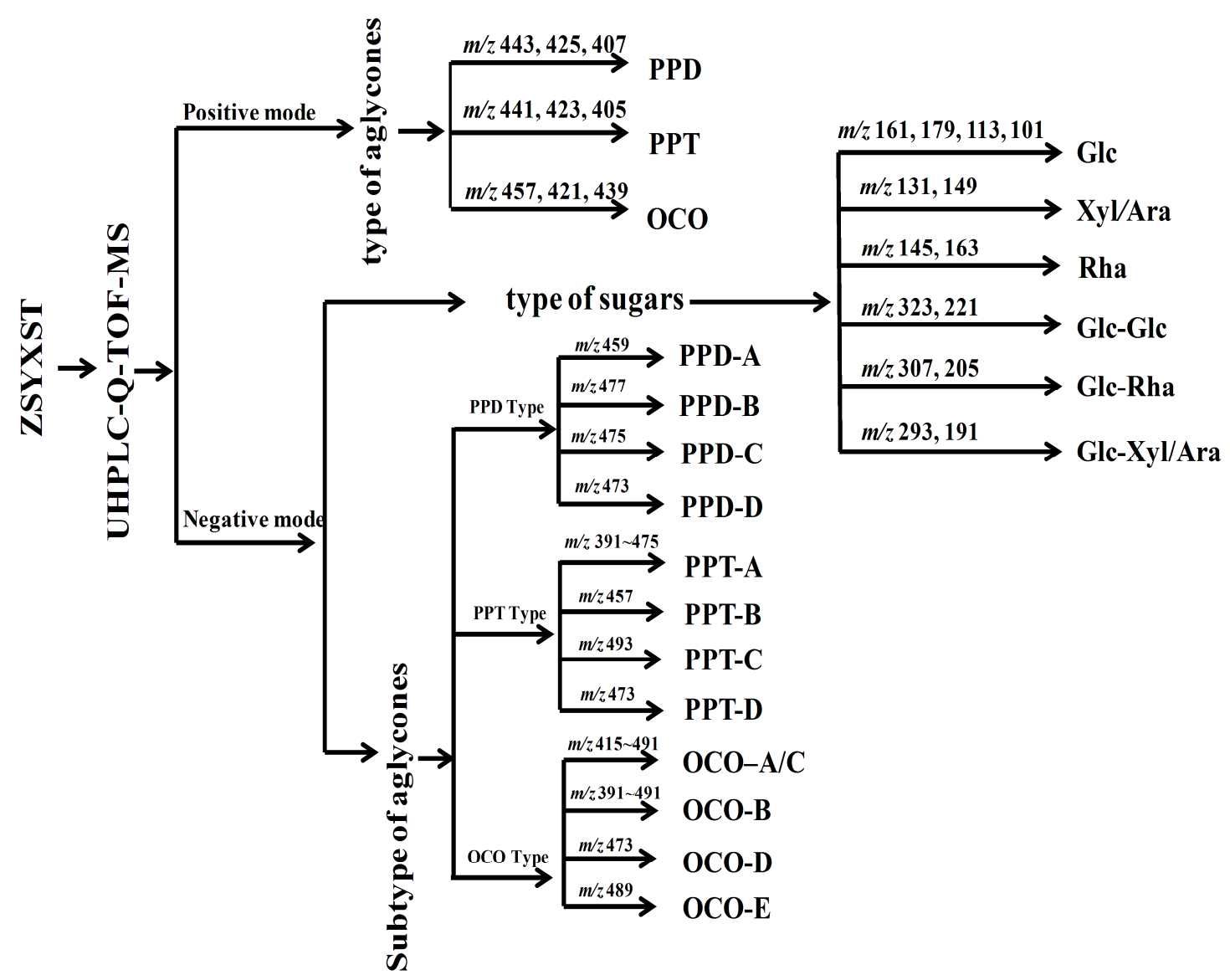

Figure 1. A diagram for rapid classification and identification of saponins by characteristic ions in MS and MS/MS mode.

\subsubsection{Differentiation of Sub-Types of Aglycone}

With the help of positive MS spectra data, the aglycone types could be deduced. However, there were several aglycone sub-types. Fortunately, those sub-types could be distinguished by characteristic fragment ions between $\mathrm{m} / \mathrm{z} 390$ to 500 in the negative MS/MS spectra. Specifically, PPD-A type could be observed at $m / z 459$, PPD-B type at $m / z 477$, PPD-C type at $m / z 475$, and PPD-D type at $m / z 457$, while the ions at $m / z$ 391-475 could be attributed to PPT-A type, $m / z 457$ to PPT-B type, $m / z 493$ to PPT-C type, $m / z 473$ to PPT-D type; in OCO types, the ions at $m / z 415-491$ belonged to OCO-A or -C type, however, a neutral loss of $180 \mathrm{Da}$ from precursor ions, it could be attributed to OCO-A type, as for OCO-B, -D, -E types, diagnostic ions at $m / z$ 391-491, 473, and 489 could be observed, respectively (Figures 1 and 2).

\subsubsection{Differentiation of Sugar Moieties}

In negative MS/MS spectra, it could be found that the terminal sugar moiety exposed to the outside in the spatial structure would cleave first. For example, in PPD type, the end sugar moiety at C-3 was cleaved first, and then the terminal sugar moiety at C-20 would cleave afterwards. However, in the PPT type, the terminal sugar moiety at C-20 was eliminated first, and then the end sugar moiety at C-6 would cleave later. OCO type saponins also conformed to this rule.

Sugar moieties linked to an aglycone through glycoside ether bonds at C-3, C-6 and C-20 or other positions, cleaved successively from the saponins. The most common sugar neutral losses were $132 \mathrm{Da}, 146 \mathrm{Da}, 162 \mathrm{Da}, 278 \mathrm{Da}, 294 \mathrm{Da}$ and $324 \mathrm{Da}$, which correspond to arabinose/xylose (Ara/Xyl), rhamnose (Rha), glucose (Glc), Rha-Ara/Xyl, Glc-Ara/Xyl and Glc-Glc, respectively. In the lower 
mass region of negative MS/MS spectra, characteristic fragment ions could be observed to further identify sugar moieties, such as $m / z$ 161, 179, 119, 113, 101 for Glc, $m / z$ 145, 163 for Rha, $m / z$ 131, 149 for Xyl or Ara, m/z 323 or 221 for Glc-Glc, m/z 307, 205 for Glc-Rha, and m/z 293,191 for Glc-Xyl/Ara (Figure 1).

In addition, in the lower mass region of positive MS spectra, characteristic fragment ions also could be observed to further authenticate sugar moieties, such as $m / z 325$ for Glc-Glc, $m / z 309$ for Glc-Rha, m/z 295 for Glc-Xyl/Ara (Table 1).

Table 1. The characteristic ions of saponins in ZSYXST.

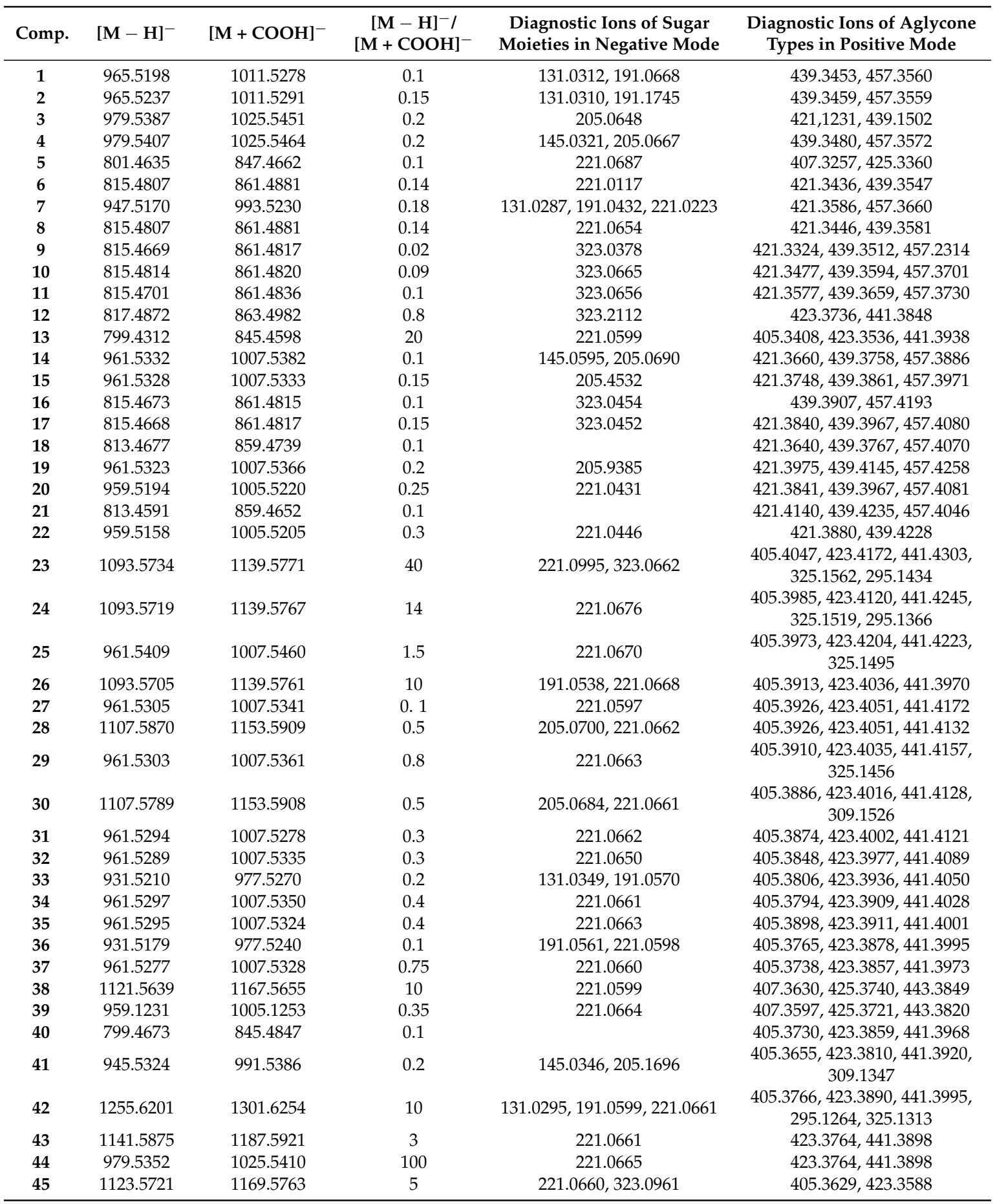


Table 1. Cont.

\begin{tabular}{|c|c|c|c|c|c|}
\hline Comp. & {$[\mathbf{M}-\mathbf{H}]^{-}$} & {$[\mathrm{M}+\mathrm{COOH}]^{-}$} & $\begin{array}{c}{\left[\mathrm{M}-\mathrm{H}^{-} /\right.} \\
{\left[\mathrm{M}+\mathrm{COOH}^{-}\right.}\end{array}$ & $\begin{array}{l}\text { Diagnostic Ions of Sugar } \\
\text { Moieties in Negative Mode }\end{array}$ & $\begin{array}{c}\text { Diagnostic Ions of Aglycone } \\
\text { Types in Positive Mode }\end{array}$ \\
\hline 46 & 901.5177 & 947.5063 & 0.88 & 191.0660 & $405.3629,423.3588,441.3587$ \\
\hline 47 & 769.3977 & 815.4032 & 0.15 & $131.0231,191.0623$ & $405.3629,423.3588$ \\
\hline 48 & 901.5008 & 947.5050 & 0.8 & 191.0601 & $405.3629,423.3588,441.3887$ \\
\hline 49 & 769.4713 & 815.4661 & 0.2 & 191.0600 & $405.3587,423.3712,441.3816$ \\
\hline 50 & 959.5032 & 1005.5082 & 0.4 & 221.0672 & $421.3554,439.3660,457.3768$ \\
\hline 51 & 1109.5496 & 1155.5567 & 2.5 & 221.0670 & $421.3541,439.3654$ \\
\hline 52 & 769.4673 & 815.4610 & 0.15 & 191.0643 & $405.3584,423.3694$ \\
\hline 53 & 961.5108 & 1007.5159 & 0.4 & 221.0667 & $405.3558,423.3683,441.3798$ \\
\hline 54 & 1123.5610 & 1169.5643 & 2.5 & 221.0661 & $405.3558,423.3683,441.3798$ \\
\hline 55 & 769.4412 & 815.4537 & 0.1 & 131.0329 & $405.3565,423.3679,441.3781$ \\
\hline 56 & 915.5043 & 961.5123 & 60 & $131.331,205.0690$ & $405.3565,423.3679,441.3781$ \\
\hline 57 & 1123.5456 & 1169.5495 & 4 & 221.0653 & $423.3781,441.3878,325.1177$ \\
\hline 58 & 1123.5427 & 1169.5475 & 4 & $221.0644,323.0924$ & $405.3552,423.3661,441.3768$ \\
\hline 59 & 1121.5226 & 1167.5242 & 5 & $221.0655,323.0970$ & $\begin{array}{c}421.3487,439.3613,457.3717 \\
325.1176\end{array}$ \\
\hline 60 & 1125.5476 & 1171.5509 & 4 & $221.0657,323.0960$ & $407.3671,425.3739$ \\
\hline 61 & 1123.5306 & 1169.5326 & 4 & 221.0651 & $405.3552,423.3649,441.3748$ \\
\hline 62 & 797.4781 & 843.4276 & 0.1 & & $421.3488,439.3595,457.3695$ \\
\hline 63 & 1123.5154 & 1169.5164 & 3 & 221.0645 & $405.3554,423.3641,441.3708$ \\
\hline 64 & 947.4713 & 993.4633 & 0.25 & $191.0598,221.0661$ & $421.3447,439.3612,457.3701$ \\
\hline 65 & 961.4723 & 1007.4753 & 0.3 & 221.0644 & $407.3661,425.3773$ \\
\hline 66 & 781.4321 & 827.42225 & 0.1 & & $405.3531,423.3637,441.3738$ \\
\hline 67 & 961.4687 & 1007.4704 & 0.2 & 221.0660 & $405.3523,423.3623,441.3754$ \\
\hline 68 & 961.4623 & 1007.4634 & 6 & 221.0664 & $405.3534,423.3665,441.3771$ \\
\hline 69 & 799.4215 & 845.4232 & 0.3 & 221.0612 & $405.3503,423.3621,441.3719$ \\
\hline 70 & 961.4620 & 1007.4617 & 0.2 & 221.0618 & $405.1917,423.3524,441.3701$ \\
\hline 71 & 961.4630 & 1007.4635 & 0.7 & 221.0660 & $405.3501,423.3633,441.3721$ \\
\hline 72 & 769.4185 & 815.4207 & 0.35 & $131.0304,191.0624$ & $405.3499,423.3609,441.3706$ \\
\hline 73 & 799.4331 & 845.4304 & 0.8 & $221.0588,323.0889$ & $\begin{array}{c}405.3489,423.3597,441.3700 \\
325.1119\end{array}$ \\
\hline 74 & 959.4537 & 1005.4554 & 0.22 & 221.0580 & $439.3546,457.3632,325.1057$ \\
\hline 75 & 1371.5895 & 1417.5705 & 100 & $\begin{array}{c}131.0298,221.0645,293.0659 \\
323.0926\end{array}$ & $\begin{array}{c}407.3640,425.3740,443.3839 \\
325.1109,295.1006\end{array}$ \\
\hline 76 & 901.4601 & 947.4621 & 0.8 & 191.0599 & $407.3629,4253724,443.3830$ \\
\hline 77 & 901.4614 & 947.4655 & 0.6 & $131.0334,191.0621$ & $407.3621,4253733,443.3843$ \\
\hline 78 & 783.4448 & 829.4493 & 0.1 & $145.0247,205.0579$ & $405.3472,423.3587,441.3686$ \\
\hline 79 & 1239.5732 & 1285.5715 & 15 & $221.0642,293.0883,323.1087$ & $\begin{array}{c}407.3631,425.3733,443.3838 \\
325.1097,295.0995\end{array}$ \\
\hline 80 & 637.3796 & 683.4054 & 0.1 & & $405.3461,423.3562,441.3665$ \\
\hline 81 & 1269.5894 & 1315.5809 & 50 & $221.0468,323.0734$ & $407.3614,425.3722,443.3799$ \\
\hline 82 & 1239.5892 & 1315.5879 & 85 & $\begin{array}{c}131.0342,191.0660,221.0661 \\
323.0979\end{array}$ & $407.3624,425.3726,443.3827$ \\
\hline 83 & 1105.5357 & 1151.5401 & 0.1 & 221.0664 & $405.3459,423.3565,441.3661$ \\
\hline 84 & 1105.5425 & 1151.5449 & 0.1 & 221.0596 & $405.3421,423.3554,441.3667$ \\
\hline 85 & 1239.2331 & 1285.2377 & 40 & $131.0121,191.0366,221.0663$ & $407.3665,425.3779,443.3760$ \\
\hline 86 & 1107.5643 & 1153.5662 & 10 & $221.0488,323.0729$ & $407.3613,425.3719,443.3813$ \\
\hline 87 & 1107.5632 & 1153.5643 & 6.6 & 221.0599 & $407.3601,425.3677,443.3805$ \\
\hline 88 & 1077.5696 & 1123.5746 & 3.3 & $131.0257,191.0466,221.0540$ & $407.3583,425.3682,443.3781$ \\
\hline 89 & 1077.5705 & 1123.5761 & 3.3 & $131.0265,191.0453,221.0536$ & $407.3583,425.3690,443.3800$ \\
\hline 90 & 943.5121 & 989.5183 & 8 & 221.0662 & $405.3433,423.3545,441.3794$ \\
\hline 91 & 945.5351 & 991.5398 & 0.3 & 221.5863 & $\begin{array}{c}407.3622,425.3730,443.3826 \\
325.1087\end{array}$ \\
\hline 92 & 945.5458 & 991.5516 & 0.35 & $221.0664,323.0721$ & $\begin{array}{c}407.3829,425.3941,443.4048 \\
325.1278\end{array}$ \\
\hline 93 & 619.4279 & 665.4239 & 0.1 & & $405.4064,423.4219,441.4347$ \\
\hline 94 & 619.4255 & 665.4245 & 0.1 & & $405.4064,423.4202,441.4332$ \\
\hline
\end{tabular}




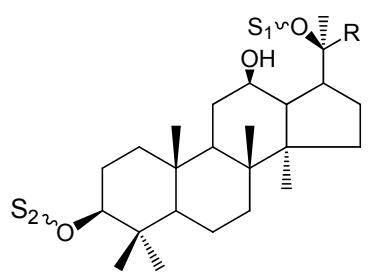

PPD-A

$\mathrm{PPD}_{1} \mathrm{C}_{1} \mathrm{R}=$

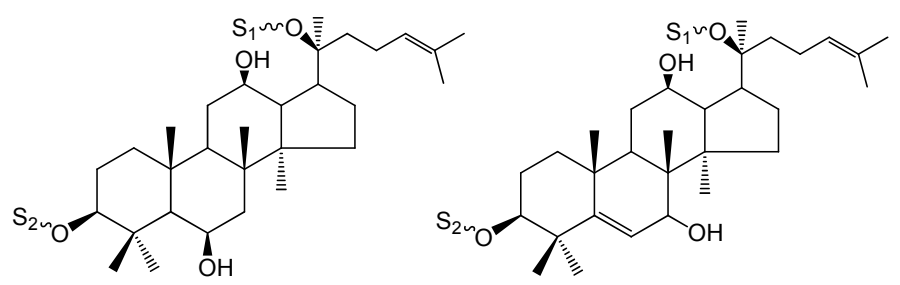

PPD-C 2

PPD-D
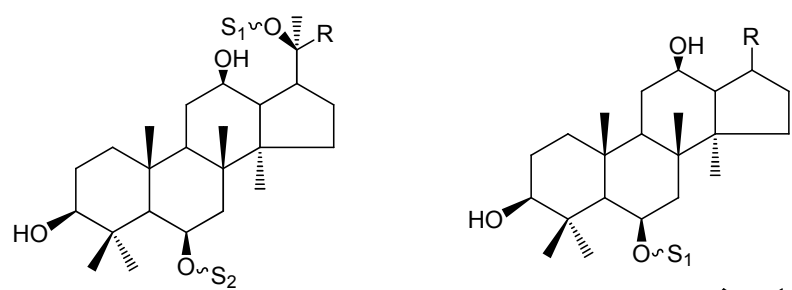

PPD-D 2<smiles></smiles><smiles>[R]=C(C)CCC=C(C)C</smiles>

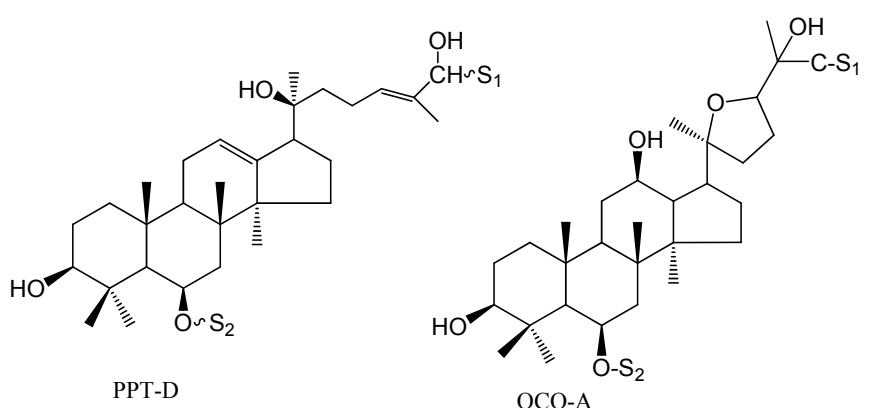

OCO-A

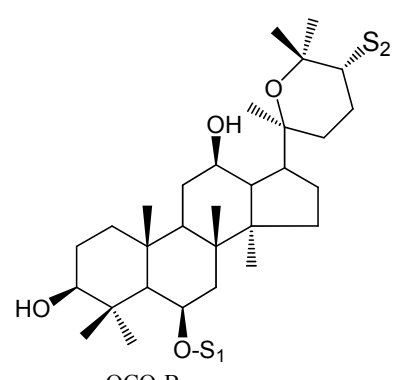

OCO-B

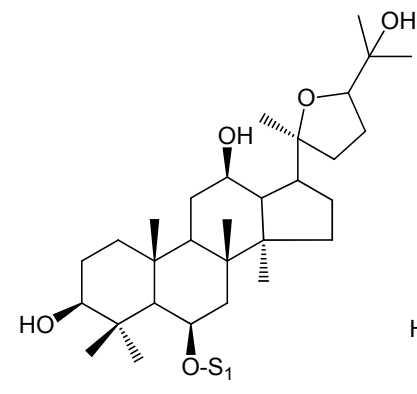

OCO-C

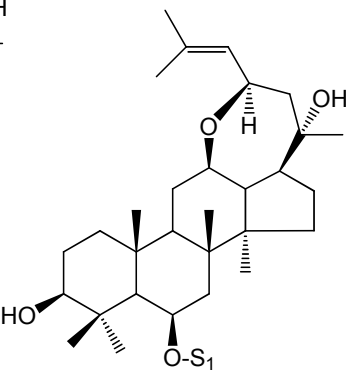

OCO-D

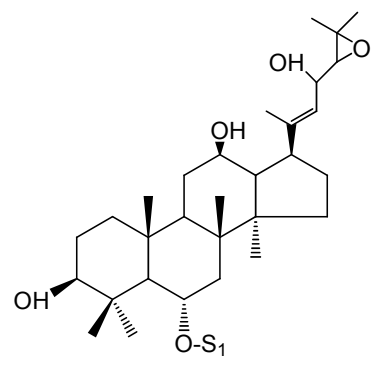

OCO-E

Figure 2. The structures of different types of saponin aglycone.

\subsubsection{Identification of Sugar Chains at $\mathrm{C}-20$ by the $[\mathrm{M}-\mathrm{H}]^{-}$to $[\mathrm{M}+\mathrm{COOH}]^{-}$Peak Ratio}

There was an interesting phenomenon in the negative MS spectra. The ratio of quasi-molecular ions $\left\{[\mathrm{M}-\mathrm{H}]^{-}\right.$to $\left.[\mathrm{M}+\mathrm{COOH}]^{-}\right\}$were related to the sugar chains at $\mathrm{C}-20$. When there were more than one sugar located at $\mathrm{C}-20$, the peak ratio of $[\mathrm{M}-\mathrm{H}]^{-}$to $[\mathrm{M}+\mathrm{COOH}]^{-}$was higher than 0.5 , however, when there was only one sugar or none linked at C-20, the peak ratio would be lower than 0.5 (Figures 3A, 4A and 5A). These characteristics could be explained by the existence of the space effect. 


\subsection{Identification of Compounds in ZSYXST}

\subsubsection{Identification of PPD Type Saponins}

In Figure 3A,B, the molecular weight of compound 91 could be deduced as 946 through the quasi-molecular ions at $\mathrm{m} / z$ 945.5351 $[\mathrm{M}-\mathrm{H}]^{-}, 991.5398[\mathrm{M}+\mathrm{COOH}]^{-}$and $969.5266[\mathrm{M}+\mathrm{Na}]^{+}$. The peak ratio of $[\mathrm{M}-\mathrm{H}]^{-}$to $[\mathrm{M}+\mathrm{COOH}]^{-}$was about 0.3 , which indicated that the number of sugar moities at C-20 was less than one (Figure 3A). Furthermore, diagnostic ions at $m / z 407,443,425$ could be observed which indicated that the saponin should be PPD Type (Figures 1 and 3B). In the MS/MS spectrum of $[\mathrm{M}-\mathrm{H}]^{-}$(Figure 3C), fragment ions at $m / z 783.4954,621.4422$ and 459.3887 could be deduced as three successive neutral losses of glucose.
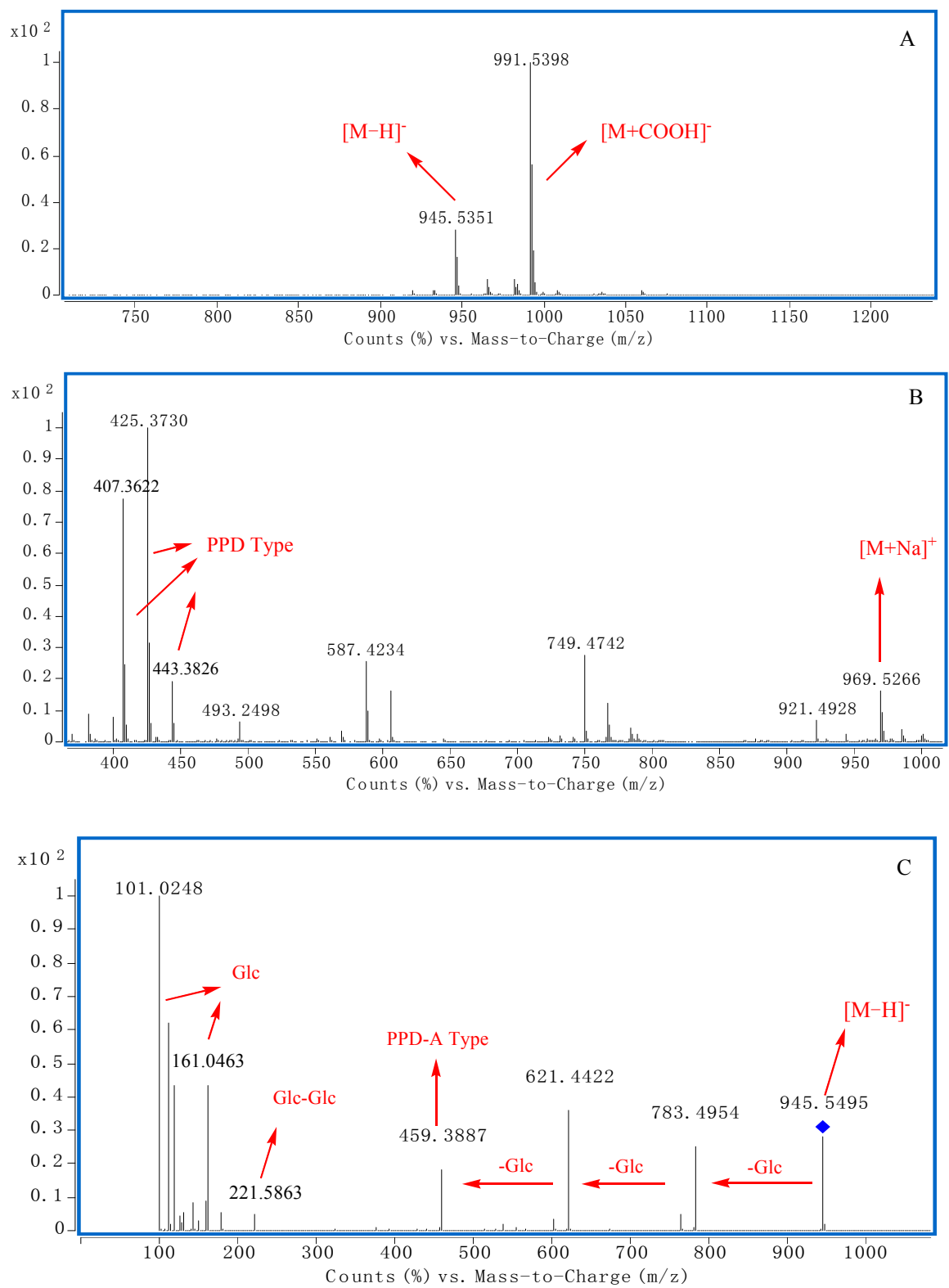

Figure 3. The typical TOF-MS spectra and fragmentation pathways of compound 91. (A) MS spectrum in negative mode; (B) MS spectrum in positive mode; (C) MS/MS spectrum in negative mode.

From the diagnostic ions at $m / z$ 459, the aglycone could be deduced to be PPD-A type (Figures 1 and 2). Fragment ions at $m / z 221$ indicated there was a Glc-Glc chain linked to the aglycone. 
According to the above diagnostic ions and comparison of the retention time with a reference, 91 could unambiguously be identified as ginsenoside Rd. Similar fragment ions were observed in 92.

Compound 75 produced $[\mathrm{M}-\mathrm{H}]^{-}$ions at $m / z 1371.5895$ and the peak ratio of $[\mathrm{M}-\mathrm{H}]^{-}$to $[\mathrm{M}+\mathrm{COOH}]^{-}$was 100 in negative mode. In MS/MS mode, diagnostic fragment ions of sugar neutral loss at $m / z 1107.5948[\mathrm{M}-\mathrm{H}-132-132]^{-}, 945.5704[\mathrm{M}-\mathrm{H}-132-132-162]^{-}, 783.4893$ $[\mathrm{M}-\mathrm{H}-132-132-162-162]^{-}, 459.5118[\mathrm{M}-\mathrm{H}-132-132-162-162-162-162]^{-}$and Glc-Glc diagnostic fragment ions at $m / z 221.0645,293.0659,323.0926$ were observed. In positive mode, $\mathrm{m} / \mathrm{z}$ 407.3640, 425.3740, 443.3839 indicate 75 belongs to the PPD type. According to the above MS cleavage rules and the literature [17], compound 75 was easily identified as notoginsenoside D.

Compound 88 generated $[\mathrm{M}-\mathrm{H}]^{-}$and $[\mathrm{M}+\mathrm{COOH}]^{-}$ions at $\mathrm{m} / z 1077.5696$ and 1123.5746, the peak ratio of $[\mathrm{M}-\mathrm{H}]^{-}$to $[\mathrm{M}+\mathrm{COOH}]^{-}$was 3.3 in negative mode. PPD Type diagnostic ions at $m / z$ 407.3583, 425.3682, 443.3781 were obtained in positive mode. According to negative MS/MS diagnostic fragment ions, such as $m / z 945.4812[\mathrm{M}-\mathrm{H}-132]^{-}, 783.4405[\mathrm{M}-\mathrm{H}-132-162]^{-}$, $621.4014[\mathrm{M}-\mathrm{H}-132-162-162]^{-}, 459.3421[\mathrm{M}-\mathrm{H}-132-162-162-162]^{-}$, as well as literature data [12], 88 was identified as notoginsenoside L. Similar fragment ions were observed in compound 89.

In the positive MS spectrum, compound 60 produced ions at $m / z 407.3671,425.3739$ which could be used to identify it as PPD type. In the negative MS spectrum, the peak ratio of $[\mathrm{M}-\mathrm{H}]^{-}$ $(\mathrm{m} / \mathrm{z} 1125.5476)$ and $[\mathrm{M}+\mathrm{COOH}]^{-}(\mathrm{m} / \mathrm{z}$ 1171.5509) was about 4 , which indicated that there was more than one sugar linked at C-20. In the MS/MS spectrum, characteristic fragment ions at $\mathrm{m} / \mathrm{z} 963.5411$ $[\mathrm{M}-\mathrm{H}-162]^{-}, 801.4900[\mathrm{M}-\mathrm{H}-162-162]^{-}, 635.4389[\mathrm{M}-\mathrm{H}-162-162-162]^{-}, 477.3889$ [M $-\mathrm{H}-162-162-162-162]^{-}$, Glc-Glc diagnostic fragment ions at $\mathrm{m} / \mathrm{z} 221.0657,323.0960$, and PPD-B type characteristic fragment ions at $m / z 477.3889$ were all observed. According to the MS

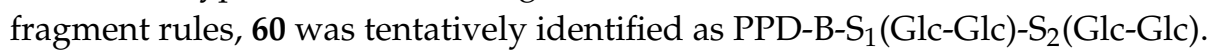

Similarly, with the help of MS cleavage rules, reference compounds and literature data, another eight PPD type saponins were identified. Thus compounds 5, 38, 39, 79, 81, 82, 85, 86, 87 were identified or tentatively identified as ginsenoside $C-Y_{1}$, quinquenoside $I V$, notoginsenoside $G$, chikusetsusaponin VI, ginsenoside $\mathrm{Ra}_{0}$ /quinquenoside $\mathrm{V}$, notoginsenoside $\mathrm{Fa}$, ginsenoside $\mathrm{Ra}_{3}$, ginsenoside $R b_{1}$, isomer of ginsenoside $R b_{1}$, respectively (Tables 1 and 2).

\subsubsection{Identification of PPT Type Saponins}

According to the quasi-molecular ions at $m / z 769.4185[\mathrm{M}-\mathrm{H}]^{-}, 815.4207[\mathrm{M}+\mathrm{COOH}]^{-}$and $793.4671[\mathrm{M}+\mathrm{Na}]^{+}$, the molecular weight of compound 72 should be 770 (Figure 4A,B). The peak ratio of $[\mathrm{M}-\mathrm{H}]^{-}$to $[\mathrm{M}+\mathrm{COOH}]^{-}$was about 0.35 , which indicated that there was less than one sugar linked at C-20 (Figure 4A). Furthermore, the aglycone type could be identified as PPT Type through diagnostic ions at $m / z 405,441,423$ (Figures 1 and 4B). In the MS/MS spectrum of $[\mathrm{M}-\mathrm{H}]^{-}$ (Figure 4C), fragment ions at $\mathrm{m} / \mathrm{z} 637.4336,475.3814$ could be deduced to represent neutral losses of xylose and glucose successively or simultaneously. Fragment ions at $m / z 191$ indicated there was a Glc-Xyl chain in 72. Characteristic ions at $\mathrm{m} / z 391$ and 475 indicated the aglycone should be PPT-A type (Figures 1 and $4 \mathrm{C}$ ). According to the peak ratio rule of $[\mathrm{M}-\mathrm{H}]^{-}$to $[\mathrm{M}+\mathrm{COOH}]^{-}$, we could deduce that the Glc-Xyl chain should be linked at the C-6 position of the aglycone. As a result, compound 72 could be identified as notoginsenoside $\mathrm{R}_{2}$.

Compound 23 produced $[\mathrm{M}-\mathrm{H}]^{-}$ions at $\mathrm{m} / \mathrm{z} 1093.5734$ in negative mode, while the peak ratio of $[\mathrm{M}-\mathrm{H}]^{-}$to $[\mathrm{M}+\mathrm{COOH}]^{-}$was 40 . In MS/MS mode, characteristic fragment ions at $\mathrm{m} / \mathrm{z} 961.5389$ $[\mathrm{M}-\mathrm{H}-132]^{-}, 637.4333[\mathrm{M}-\mathrm{H}-132-162-162]^{-}, 475.3832[\mathrm{M}-\mathrm{H}-132-162-162-162]^{-}$, 391.2772 and Glc-Glc diagnostic fragment ions at $m / z 221.0995,323.0662$ were obtained. In positive mode, it produced ions at $m / z 405.4047,423.4172,441.4303$ which could be used to identify it as PPT-A type. According to the retention time and MS fragment rules, $\mathbf{2 3}$ was unambiguously identified as sanchirhinoside $A_{6}$. Similar diagnostic fragment ions were observed in compounds $\mathbf{2 4}$ and $\mathbf{2 6 .}$ 
Table 2. Identification of compounds in ZSYXST.

\begin{tabular}{|c|c|c|c|c|c|}
\hline Comp. & Rt (min) & & MS/MS $m / z$ & Area $\%$ & Identification \\
\hline 1 & 7.54 & 965.5198 & $785.9465[\mathrm{M}-\mathrm{H}-180]^{-}, 653.3969[\mathrm{M}-\mathrm{H}-180-132]^{-}, 491.3843[\mathrm{M}-\mathrm{H}-180-132-162]^{-}, 415.3350$ & 0.019 & 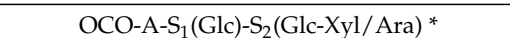 \\
\hline 2 & 8.611 & 965.5237 & $785.9489[\mathrm{M}-\mathrm{H}-180]^{-}, 653.3794[\mathrm{M}-\mathrm{H}-180-132]^{-}, 491.3654[\mathrm{M}-\mathrm{H}-180-132-162]^{-}, 415.3229$ & 0.006 & isomer of OCO-A-S $\left(\right.$ Glc)-S $S_{2}($ Glc-Xyl/Ara) * \\
\hline 3 & 8.811 & 979.5387 & $799.4633[\mathrm{M}-\mathrm{H}-180]^{-,}, 635.3669[\mathrm{M}-\mathrm{H}-180-146]^{-}, 491.3370[\mathrm{M}-\mathrm{H}-180-146-162]^{-}, 415.3301$ & 0.016 & OCO-A-S S $_{1}$ Glc)-S $S_{2}$ (Glc-Rha) [14] \\
\hline 4 & 10.189 & 979.5407 & $799.4532[\mathrm{M}-\mathrm{H}-180]^{-}, 635.3868[\mathrm{M}-\mathrm{H}-180-146]^{-}, 491.3569[\mathrm{M}-\mathrm{H}-180-146-162]^{-}, 415.3277$ & 0.006 & isomer of OCO-A-S $\mathrm{S}_{1}(\mathrm{Glc})-\mathrm{S}_{2}$ (Glc-Rha) [14] \\
\hline 5 & 12.992 & 801.4635 & $639.4097[\mathrm{M}-\mathrm{H}-162]^{-}, 477.3605[\mathrm{M}-\mathrm{H}-162-162]^{-}$ & 0.006 & ginsenoside $C-Y_{1}[22]$ \\
\hline 6 & 13.699 & 815.4807 & $653.4212[\mathrm{M}-\mathrm{H}-162]^{-}, 491.3770[\mathrm{M}-\mathrm{H}-162-162]^{-}, 391.2872$ & 0.032 & OCO-B-S $\mathrm{S}_{1}(\mathrm{Glc}-\mathrm{Glc})-\mathrm{S}_{2}(\mathrm{OH})[23]$ \\
\hline 7 & 13.852 & 947.5170 & $785.4339[\mathrm{M}-\mathrm{H}-162]^{-}, 623.4062[\mathrm{M}-\mathrm{H}-162-162]^{-}, 491.4131[\mathrm{M}-\mathrm{H}-162-162-132]^{-}, 415.3310$ & 0.032 & vinaginsenoside $R_{5}$ or yesanchinoside $C[24,25]$ \\
\hline 8 & 14.151 & 815.4801 & $653.4276[\mathrm{M}-\mathrm{H}-162]^{-}, 491.3788[\mathrm{M}-\mathrm{H}-162-162]^{-}, 391.2878$ & 0.032 & isomer of OCO-B-S $\mathrm{S}_{1}(\mathrm{Glc}-\mathrm{Glc})-\mathrm{S}_{2}(\mathrm{OH})$ \\
\hline 9 & 14.252 & 861.4892 & $653.4263[\mathrm{M}-\mathrm{H}-162]^{-}, 491.3777[\mathrm{M}-\mathrm{H}-162-162]^{-}, 415.3253$ & 0.010 & majonoside $R_{1}[12,19]$ \\
\hline 10 & 14.582 & 815.4814 & $653.4162[\mathrm{M}-\mathrm{H}-162]^{-}, 491.3716[\mathrm{M}-\mathrm{H}-162-162]^{-}, 415.3533$ & 0.019 & isomer of majonoside $\mathrm{R}_{1}[12,19]$ \\
\hline 11 & 15.03 & 815.4701 & $653.4162[\mathrm{M}-\mathrm{H}-162]^{-}, 491.3716[\mathrm{M}-\mathrm{H}-162-162]^{-}, 415.3519$ & 0.151 & isomer of majonoside $R_{1}[12,19]$ \\
\hline 12 & 15.948 & 817.4872 & $655.4462[\mathrm{M}-\mathrm{H}-162]^{-}, 493.3934[\mathrm{M}-\mathrm{H}-162-162]^{-}$ & 0.048 & 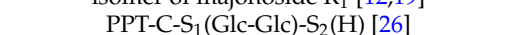 \\
\hline 13 & 16.431 & 799.4312 & $799.4678[\mathrm{M}-\mathrm{H}-162]^{-}, 637.4793[\mathrm{M}-\mathrm{H}-162-162]^{-}, 475.3866[\mathrm{M}-\mathrm{H}-162-162-162]^{-}, 391.2904$ & 0.013 & ginsenoside Rf [27] \\
\hline 14 & 16.714 & 961.5332 & $799.4679[\mathrm{M}-\mathrm{H}-162]^{-}, 653.4002[\mathrm{M}-\mathrm{H}-162-146]^{-}, 491.3304[\mathrm{M}-\mathrm{H}-162-146-162]^{-}, 391.2772$ & 0.032 & OCO-B-S $S_{1}\left(\right.$ Glc-Rha-Glc)-S $\mathrm{S}_{2}\left(\mathrm{CH}_{3}\right)[26]$ \\
\hline 15 & 17.597 & 961.5328 & $799.5297[\mathrm{M}-\mathrm{H}-162]^{-}, 653.4002[\mathrm{M}-\mathrm{H}-162-146]^{-}, 491.3693[\mathrm{M}-\mathrm{H}-162-146-162]^{-}, 391.8943$ & 0.032 & isomer of OCO-B-S $\mathrm{S}_{1}$ (Glc-Rha-Glc)- $\mathrm{S}_{2}\left(\mathrm{CH}_{3}\right)$ \\
\hline 16 & 18.398 & 815.4868 & $653.4263[\mathrm{M}-\mathrm{H}-162]^{-}, 491.3777[\mathrm{M}-\mathrm{H}-162-162]^{-}, 415.3361$ & 0.515 & isomer of majonoside $R_{1}[12,19]$ \\
\hline 17 & 18.092 & 815.4673 & $653.4373[\mathrm{M}-\mathrm{H}-162]^{-}, 491.3788[\mathrm{M}-\mathrm{H}-162-162]^{-}, 415.3415$ & 0.032 & isomer of majonoside $\mathrm{R}_{1}[12,19]$ \\
\hline 18 & 19.481 & 859.4723 & $651.4111[\mathrm{M}-\mathrm{H}-162]^{-}, 489.3579[\mathrm{M}-\mathrm{H}-162-162]^{-}$ & 0.019 & OCO-E-S $($ Glc-Glc) * \\
\hline 19 & 20.247 & 961.5323 & $799.4824[\mathrm{M}-\mathrm{H}-162]^{-}, 653.4002[\mathrm{M}-\mathrm{H}-162-146]^{-}, 491.3558[\mathrm{M}-\mathrm{H}-162-146-162]^{-}, 391.3441$ & 0.016 & isomer of OCO-B-S $\mathrm{S}_{1}$ (Glc-Rha-Glc)- $\mathrm{S}_{2}\left(\mathrm{CH}_{3}\right)$ [21] \\
\hline 20 & 21.142 & 959.5194 & $797.4207[\mathrm{M}-\mathrm{H}-162]^{-}, 635.3567[\mathrm{M}-\mathrm{H}-162-162]^{-}, 473.3460[\mathrm{M}-\mathrm{H}-162-162-162]^{-}$ & 0.016 & OCO-D-S S $_{1}$ (Glc-Glc-Glc) * \\
\hline 21 & 22.39 & 859.4721 & $651.4113[\mathrm{M}-\mathrm{H}-162]^{-,}, 489.3635[\mathrm{M}-\mathrm{H}-162-162]^{-}{ }^{\prime}$ & 0.023 & isomer of OCO-E-S 1 (Glc-Glc) * \\
\hline 22 & 23.909 & 959.5158 & $797.4575[\mathrm{M}-\mathrm{H}-162]^{-}, 635.4076[\mathrm{M}-\mathrm{H}-162-162]^{-}, 473.3611[\mathrm{M}-\mathrm{H}-162-162-162]^{-}$ & 0.016 & isomer of OCO-D-S ${ }_{1}($ Glc-Glc-Glc) * \\
\hline 23 & 26.936 & 1093.5734 & $961.5389[\mathrm{M}-\mathrm{H}-132]^{-}, 637.4333[\mathrm{M}-\mathrm{H}-132-162-162]^{-}, 475.3832[\mathrm{M}-\mathrm{H}-132-162-162-162]^{-}, 391.2872$ & 0.093 & sanchirhinoside $\mathrm{A}_{6}$ \\
\hline 24 & 28.314 & 1093.5719 & $961.5374[\mathrm{M}-\mathrm{H}-132]^{-}, 637.4382[\mathrm{M}-\mathrm{H}-132-162-162]^{-}, 475.3753[\mathrm{M}-\mathrm{H}-132-162-162-162]^{-}, 391.2883$ & 0.064 & isomer of sanchirhinoside $A_{6}[19,28]$ \\
\hline 25 & 28.962 & 961.5374 & $799.4883[\mathrm{M}-\mathrm{H}-162]^{-}, 637.4389[\mathrm{M}-\mathrm{H}-162-162]^{-}, 475.3826[\mathrm{M}-\mathrm{H}-162-162-162]^{-}, 391.2872$, & 0.328 & $\begin{array}{l}\text { notoginsenoside } R_{3} \\
\text { nots }\end{array}$ \\
\hline 26 & 29.751 & 1093.5705 & $961.5272[\mathrm{M}-\mathrm{H}-132]^{-}, 637.4367[\mathrm{M}-\mathrm{H}-132-162-162]^{-}, 475.3753[\mathrm{M}-\mathrm{H}-132-162-162-162]^{-}, 391.2879$ & 0.028 & isomer of sanchirhinoside $\mathrm{A}_{6}[19,28]$ \\
\hline 27 & 30.175 & 961.5305 & $799.4817[\mathrm{M}-\mathrm{H}-162]^{-}, 637.4185[\mathrm{M}-\mathrm{H}-162-162]^{-}, 475.3757[\mathrm{M}-\mathrm{H}-162-162-162]^{-}, 391.2901$ & 0.187 & notoginsenoside $\mathrm{N}$ \\
\hline 28 & 30.175 & 1107.5870 & $961.5311[\mathrm{M}-\mathrm{H}-146]^{-}, 799.4749[\mathrm{M}-\mathrm{H}-146-162]^{-}, 637.4310[\mathrm{M}-\mathrm{H}-146-162-162]^{-}$ & - & yesanchinoside E [17] \\
\hline 29 & 30.599 & 961.5303 & $\begin{array}{l}475.3794[\mathrm{M}-\mathrm{H}-146-162-162-162]^{-}, 391.2892 \\
637.4328[\mathrm{M}-\mathrm{H}-162-162]^{-}, 475.3781[\mathrm{M}-\mathrm{H}-162-162-162]^{-}, 391.2912\end{array}$ & 0.170 & notoginsenoside $R_{6}$ \\
\hline 30 & 30.999 & 1107.5789 & $961.5299[\mathrm{M}-\mathrm{H}-146]^{-}, 799.4747[\mathrm{M}-\mathrm{H}-146-162]^{-}, 637.4224[\mathrm{M}-\mathrm{H}-146-162-162]^{-}$, & 0.026 & isomer of yesanchinoside E [17] \\
\hline 31 & 31.788 & 961.5294 & $\begin{array}{l}475.3760[\mathrm{M}-\mathrm{H}-146-162-162-162]^{-}, 391.2902 \\
799.4784[\mathrm{M}-\mathrm{H}-162]^{-}, 637.4286[\mathrm{M}-\mathrm{H}-162-162]^{-}, 475.3782[\mathrm{M}-\mathrm{H}-162-162-162]^{-}, 391.2899\end{array}$ & 0.715 & 20-O-glucoginsenoside Rf \\
\hline 32 & 32.259 & 961.5289 & $799.4824[\mathrm{M}-\mathrm{H}-162]^{-}, 637.4403[\mathrm{M}-\mathrm{H}-162-162]^{-}, 475.3832[\mathrm{M}-\mathrm{H}-162-162-162]^{-}, 391.2876$ & 0.052 & isomer of 20-O-glucoginsenoside $\mathrm{Rf}$ \\
\hline 33 & 33.661 & 931.5210 & $637.4369[\mathrm{M}-\mathrm{H}-132-162]^{-}, 475.3824[\mathrm{M}-\mathrm{H}-132-162-162]^{-}, 391.3270$ & 13.39 & notoginsenoside $R_{1}$ \\
\hline 34 & 34.296 & 961.5297 & $799.4803[\mathrm{M}-\mathrm{H}-162]^{-}, 637.4268[\mathrm{M}-\mathrm{H}-162-162]^{-}, 475.3785[\mathrm{M}-\mathrm{H}-162-162-162]^{-}, 391.2913$ & 0.151 & notoginsenoside $\mathrm{M}$ or $\mathrm{N}[19,21]$ \\
\hline 35 & 34.991 & 961.5295 & $799.4811[\mathrm{M}-\mathrm{H}-162]^{-}, 637.4194[\mathrm{M}-\mathrm{H}-162-162]^{-}, 475.3634[\mathrm{M}-\mathrm{H}-162-162-162]^{-}, 391.2920$ & 0.067 & isomer of notoginsenoside $\mathrm{M}$ or $\mathrm{N}[19,21]$ \\
\hline 36 & 35.392 & 931.5179 & $637.4281[\mathrm{M}-\mathrm{H}-132-162]^{-}, 475.3762[\mathrm{M}-\mathrm{H}-132-162-162]^{-}, 391.2901$ & 0.138 & gypenoside LXIV [29] \\
\hline
\end{tabular}


Table 2. Cont

\begin{tabular}{|c|c|c|c|c|c|}
\hline Comp. & Rt (min) & & MS/MS $m / z$ & Area\% & Identification \\
\hline 37 & 35.957 & 961.5277 & $799.4883[\mathrm{M}-\mathrm{H}-162]^{-}, 637.4255[\mathrm{M}-\mathrm{H}-162-162]^{-}, 475.3787[\mathrm{M}-\mathrm{H}-162-162-162]^{-}, 391.2877$ & 0.041 & isomer of notoginsenoside $R_{3}$ or isomer of \\
\hline 38 & 36.31 & 1121.5639 & $959.3973[\mathrm{M}-\mathrm{H}-162]^{-}, 797.4040[\mathrm{M}-\mathrm{H}-162-162]^{-}, 473.3085[\mathrm{M}-\mathrm{H}-162-162-162-162]^{-}$ & 0.229 & quinquenoside IV [27] \\
\hline 39 & 36.31 & 959.1231 & $797.3990[\mathrm{M}-\mathrm{H}-162]^{-}, 635.4110[\mathrm{M}-\mathrm{H}-162-162]^{-}, 473.3675[\mathrm{M}-\mathrm{H}-162-162-162]^{-}$ & - & notoginsenoside $\mathrm{G}$ \\
\hline 40 & 36.781 & 799.4673 & $637.4352[\mathrm{M}-\mathrm{H}-162]^{-}, 475.3815[\mathrm{M}-\mathrm{H}-162-162]^{-}, 391.2930$ & 27.59 & ginsenoside $\mathrm{Rg}_{1}$ [27] \\
\hline 41 & 37.3 & 945.5324 & $783.4775[\mathrm{M}-\mathrm{H}-162]^{-}, 637.4309[\mathrm{M}-\mathrm{H}-162-146]^{-}, 475.3791[\mathrm{M}-\mathrm{H}-162-146-162]^{-}, 391.3001$ & 7.670 & ginsenoside Re \\
\hline 42 & 37.676 & 1255.6201 & $\begin{array}{l}1123.1027[\mathrm{M}-\mathrm{H}-132]^{-}, 961.2952[\mathrm{M}-\mathrm{H}-132-162]^{-}, 799.3770[\mathrm{M}-\mathrm{H}-132-162-162]^{-}, \\
637.4266[\mathrm{M}-\mathrm{H}-132-162-162-162]^{-}, 475.3701[\mathrm{M}-\mathrm{H}-132-162-162-162-162]^{-}, 391.2900\end{array}$ & 0.032 & PPT-A-S 1 (Glc-Glc-Xyl/Ara)-S (Glc-Glc) [21] \\
\hline 43 & 39.349 & 1141.5875 & $817.4870[\mathrm{M}-\mathrm{H}-162-162]^{-}, 655.4351[\mathrm{M}-\mathrm{H}-162-162-162]^{-}, 493.3991[\mathrm{M}-\mathrm{H}-162-162-162-162]^{-}$ & 0.222 & quinquenoside $\mathrm{L}_{16}$ [18] \\
\hline 44 & 39.349 & 979.5352 & $817.4912[\mathrm{M}-\mathrm{H}-162]^{-}, 655.4429[\mathrm{M}-\mathrm{H}-162-162]^{-}, 493.4046[\mathrm{M}-\mathrm{H}-162-162-162]^{-}$ & - & PPT-C-S 1 (Glc)-S (Glc-Glc) [30] \\
\hline 45 & 41.41 & 1123.5721 & $\begin{array}{l}961.5210[\mathrm{M}-\mathrm{H}-162]^{-}, 799.4757[\mathrm{M}-\mathrm{H}-162-162]^{-}, 637.4238[\mathrm{M}-\mathrm{H}-162-162-162]^{-}, \\
475.3770[\mathrm{M}-\mathrm{H}-162-162-162-162]^{-}, 391.2870\end{array}$ & 0.248 & PPT-A-S 1 (Glc-Glc)-S $S_{2}$ (Glc-Glc) [31] \\
\hline 46 & 41.41 & 901.5177 & $637.4241[\mathrm{M}-\mathrm{H}-132-132]^{-}, 475.3827[\mathrm{M}-\mathrm{H}-132-132-162]^{-}, 391.2881$ & - & chikusetsusaponin $\mathrm{L}_{5}$ [27] \\
\hline 47 & 41.41 & 769.3977 & $637.3997[\mathrm{M}-\mathrm{H}-162]^{-}, 475.3876[\mathrm{M}-\mathrm{H}-162-132]^{-}, 391.2851$ & - & pseudoginsenoside $\mathrm{Rt}_{3}$ \\
\hline 48 & 42.717 & 901.5008 & $637.4230[\mathrm{M}-\mathrm{H}-132-132]^{-}, 475.3744[\mathrm{M}-\mathrm{H}-132-132-162]^{-}, 391.2901$ & 0.077 & isomer of chikusetsusaponin $\mathrm{L}_{5}$ [27] \\
\hline 49 & 43.223 & 769.4713 & $637.4342[\mathrm{M}-\mathrm{H}-132]^{-}, 475.3828[\mathrm{M}-\mathrm{H}-132-162]^{-}, 391.2873$ & 0.090 & notoginsenoside $R_{2}[19]$ \\
\hline 50 & 43.8 & 959.5032 & $797.4575[\mathrm{M}-\mathrm{H}-162]^{-}, 635.4076[\mathrm{M}-\mathrm{H}-162-162]^{-}, 473.3611[\mathrm{M}-\mathrm{H}-162-162-162]^{-}$ & 0.145 & isomer of OCO-D-S (Glc-Glc-Glc)* $^{*}$ \\
\hline 51 & 44.377 & 1109.5496 & $\begin{array}{l}785.4931[\mathrm{M}-\mathrm{H}-162-162]^{-}, 623.4047[\mathrm{M}-\mathrm{H}-162-162-162]^{-}, 491.3606[\mathrm{M}-\mathrm{H}-162-162-162-132]^{-}, \\
391.8654\end{array}$ & 0.032 & OCO-B-S ${ }_{1}(\mathrm{Xyl}-\mathrm{Glc}-\mathrm{Glc}-\mathrm{Glc})-\mathrm{S}_{2}(\mathrm{OH})^{*}$ \\
\hline 52 & 44.79 & 769.4673 & $637.4342[\mathrm{M}-\mathrm{H}-132]^{-}, 475.3828[\mathrm{M}-\mathrm{H}-132-162]^{-}, 391.2890$ & 0.045 & isomer of notoginsenos \\
\hline 53 & 45.108 & 961.5108 & $799.4836[\mathrm{M}-\mathrm{H}-162]^{-}, 637.4239[\mathrm{M}-\mathrm{H}-162-162]^{-}, 475.3783[\mathrm{M}-\mathrm{H}-162-162-162]^{-}, 391.2903$ & 0.174 & isomer of 20-O-glucoginseno \\
\hline 54 & 45.108 & 1123.5610 & $\begin{array}{l}961.5215[\mathrm{M}-\mathrm{H}-162]^{-}, 799.4657[\mathrm{M}-\mathrm{H}-162-162]^{-}, 637.4338[\mathrm{M}-\mathrm{H}-162-162-162]^{-} \\
475.3770[\mathrm{M}-\mathrm{H}-162-162-162-162]^{-}, 391.2891\end{array}$ & - & isomer of PPT-A-S S $_{1}$ Glc-Glc)-S ${ }_{2}$ (Glc-Glc) [31] \\
\hline 55 & 46.344 & 769.4412 & $637.4341[\mathrm{M}-\mathrm{H}-132]^{-}, 475.3807[\mathrm{M}-\mathrm{H}-132-162]^{-}, 391.2891$ & 0.196 & sanchirhinoside $\mathrm{A}_{3}[19]$ \\
\hline 56 & 46.344 & 915.5043 & $783.4801[\mathrm{M}-\mathrm{H}-132]^{-}, 637.4801[\mathrm{M}-\mathrm{H}-132-146]^{-}, 475.3756[\mathrm{M}-\mathrm{H}-132-146-162]^{-}, 391.3001$ & - & PPT-A-S 1 (Rha-Xyl)-S $($ Glc) [31] \\
\hline 57 & 46.909 & 1123.5456 & $\begin{array}{l}961.5244[\mathrm{M}-\mathrm{H}-162]^{-}, 799.4729[\mathrm{M}-\mathrm{H}-162-162]^{-}, 637.4283[\mathrm{M}-\mathrm{H}-162-162-162]^{-}, \\
475.3721[\mathrm{M}-\mathrm{H}-162-162-162-162]^{-}, 391.2911\end{array}$ & 0.051 & isomer of PPT-A-S S $_{1}$ Glc-Glc)-S $\mathrm{S}_{2}$ (Glc-Glc) [31] \\
\hline 58 & 47.169 & 1123.5427 & $\begin{array}{l}961.5227[\mathrm{M}-\mathrm{H}-162]^{-}, 799.4758[\mathrm{M}-\mathrm{H}-162-162]^{-}, 637.4274[\mathrm{M}-\mathrm{H}-162-162-162]^{-}, \\
475.3728[\mathrm{M}-\mathrm{H}-162-162-162-162]^{-}, 391.2893\end{array}$ & 0.012 & isomer of PPT-A-S $S_{1}(G l c-G l c)-S_{2}(G l c-G l c)[31]$ \\
\hline 59 & 47.84 & 1121.5226 & $\begin{array}{l}959.5057[\mathrm{M}-\mathrm{H}-162]^{-}, 797.4549[\mathrm{M}-\mathrm{H}-162-162]^{-}, 635.4088[\mathrm{M}-\mathrm{H}-162-162-162]^{-}, \\
473.3567[\mathrm{M}-\mathrm{H}-162-162-162-162]^{-}\end{array}$ & 0.077 & OCO-D-S 1 (Glc-Glc-Glc-Glc) * \\
\hline 60 & 48.17 & 1125.5476 & $\begin{array}{l}963.5411[\mathrm{M}-\mathrm{H}-162]^{-}, 801.4900[\mathrm{M}-\mathrm{H}-162-162]^{-}, 635.4389[\mathrm{M}-\mathrm{H}-162-162-162]^{-}, \\
477.3889[\mathrm{M}-\mathrm{H}-162-162-162-162]^{-}\end{array}$ & 0.066 & PPD-B-S 1 (Glc-Glc)-S $S_{2}$ (Glc-Glc) [34] \\
\hline 61 & 48.594 & 1123.5306 & $\begin{array}{l}961.5171[\mathrm{M}-\mathrm{H}-162]^{-}, 799.4745[\mathrm{M}-\mathrm{H}-162-162]^{-}, 637.4232[\mathrm{M}-\mathrm{H}-162-162-162]^{-}, \\
475.3754[\mathrm{M}-\mathrm{H}-162-162-162-162]^{-}, 391.2891\end{array}$ & 0.035 & isomer of PPT-A-S $\mathrm{S}_{1}(\mathrm{Glc}-\mathrm{Glc})-\mathrm{S}_{2}(\mathrm{Glc}-\mathrm{Glc})[31]$ \\
\hline 62 & 48.864 & 797.4781 & $635.4190[\mathrm{M}-\mathrm{H}-162]^{-}, 473.3695[\mathrm{M}-\mathrm{H}-162-162]^{-}$ & 0.039 & PPT-D-S 1 (Glc-Glc) [21] \\
\hline 63 & 49.63 & 1123.5254 & $\begin{array}{l}961.5203[\mathrm{M}-\mathrm{H}-162]^{-}, 799.4698[\mathrm{M}-\mathrm{H}-162-162]^{-}, 637.4431[\mathrm{M}-\mathrm{H}-162-162-162]^{-}, \\
475.3701[\mathrm{M}-\mathrm{H}-162-162-162-162]^{-}-3912973\end{array}$ & 0.097 & isomer of PPT-A-S $S_{1}$ (Glc-Glc)-S $\mathrm{S}_{2}$ (Glc-Glc) [31] \\
\hline 64 & 49.63 & 947.4713 & $815.3567[\mathrm{M}-\mathrm{H}-132]^{-}, 653.3778[\mathrm{M}-\mathrm{H}-132-162]^{-}, 491.3608[\mathrm{M}-\mathrm{H}-132-162-162]^{-}, 391.8554$ & - & OCO-B-S 1 (Glc-Glc-Xyl/Ara)-S $2(\mathrm{OH})[35]$ \\
\hline 65 & 50.101 & 961.4723 & $799.4722[\mathrm{M}-\mathrm{H}-162]^{-}, 637.4220[\mathrm{M}-\mathrm{H}-162-162]^{-}, 475.3720[\mathrm{M}-\mathrm{H}-162-162-162]^{-}$ & 0.1160 & vina-ginsenoside $\mathrm{R}_{4}$ \\
\hline 66 & 50.2 & 4321 & $619.3533[\mathrm{M}-\mathrm{H}-162]^{-}, 457.3661[\mathrm{M}-\mathrm{H}-162-162]^{-}$ & 0.032 & sanchirhinoside B \\
\hline 67 & 50.619 & 961.4687 & $799.4598[\mathrm{M}-\mathrm{H}-162]^{-}, 637.4037[\mathrm{M}-\mathrm{H}-162-162]^{-}, 475.3770[\mathrm{M}-\mathrm{H}-162-162-162]^{-}, 391.2866$ & 0.058 & isomer of 20-O-glucoginsenoside $R_{f}[32,33]$ \\
\hline
\end{tabular}


Table 2. Cont

\begin{tabular}{|c|c|c|c|c|c|}
\hline Comp. & Rt (min) & & MS/MS $m / z$ & Area\% & Identification \\
\hline 68 & 51.762 & 961.4623 & $781.4714[\mathrm{M}-\mathrm{H}-180]^{-}, 637.4232[\mathrm{M}-\mathrm{H}-180-144]^{-}, 475.3801[\mathrm{M}-\mathrm{H}-180-144-162]^{-}, 391.2909$ & 0.019 & $\begin{array}{c}\text { isomer of notoginsenoside } R_{3} \text { or isomer of } \\
\text { notoginsenoside } R_{6}[17,19]\end{array}$ \\
\hline $\begin{array}{l}69 \\
70\end{array}$ & $\begin{array}{l}52.221 \\
53.552\end{array}$ & $\begin{array}{l}799.4215 \\
961.4620\end{array}$ & $\begin{array}{l}637.4209[\mathrm{M}-\mathrm{H}-162]^{-}, 475.3722[\mathrm{M}-\mathrm{H}-162-162]^{-}, 391.2877 \\
799.4434[\mathrm{M}-\mathrm{H}-162]^{-}, 637.4263[\mathrm{M}-\mathrm{H}-162-162]^{-}, 475.4024[\mathrm{M}-\mathrm{H}-\end{array}$ & $\begin{array}{l}0.068 \\
0.039\end{array}$ & $\begin{array}{l}\text { isomer of ginsenoside } \mathrm{Rg}_{1}[36,37] \\
\text { isomer of } 20-O \text {-glucoginsenoside } \mathrm{R}_{f}[32,33]\end{array}$ \\
\hline 71 & 54.376 & 961.4630 & $799.4695[\mathrm{M}-\mathrm{H}-162]^{-}, 637.4074[\mathrm{M}-\mathrm{H}-162-162]^{-}, 475.3638[\mathrm{M}-\mathrm{H}-162-162-162]^{-}, 391.2881$ & 0.019 & $\begin{array}{c}\text { isomer of notoginsenoside } R_{3} \text { or isomer of } \\
\text { notoginsenoside } R_{6}[17,19]\end{array}$ \\
\hline 72 & 55.185 & 769.4795 & $637.4336[\mathrm{M}-\mathrm{H}-132]^{-}, 475.3814[\mathrm{M}-\mathrm{H}-132-162]^{-}, 391.2869$ & 1.572 & isomer of notoginsenoside $R_{2}$ \\
\hline 73 & 55.813 & 799.4331 & $637.4356[\mathrm{M}-\mathrm{H}-162]^{-}, 475.4315[\mathrm{M}-\mathrm{H}-162-162]^{-}, 391.2872$ & 0.206 & notoginsenoside $\mathrm{U}$ \\
\hline 74 & 56.461 & 959.4537 & $797.4608[\mathrm{M}-\mathrm{H}-162]^{-}, 635.4138[\mathrm{M}-\mathrm{H}-162-162]^{-}, 473.3590[\mathrm{M}-\mathrm{H}-162-162-162]^{-}$ & 0.026 & ginsenoside III or vinaginsenoside $R_{20}[38]$ \\
\hline 75 & 56.767 & 1371.5895 & $\begin{array}{l}1107.5948[\mathrm{M}-\mathrm{H}-132-132]^{-}, 945.5704[\mathrm{M}-\mathrm{H}-132-132-162]^{-}, 783.4893[\mathrm{M}-\mathrm{H}-132-132-162-162]^{-}, \\
459.5118[\mathrm{M}-\mathrm{H}-132-132-162-162-162-162]^{-}\end{array}$ & 0.296 & notoginsenoside D [17] \\
\hline 76 & 57.992 & 901.4601 & $769.4200[\mathrm{M}-\mathrm{H}-132]^{-}, 637.4355[\mathrm{M}-\mathrm{H}-132-132]^{-}, 475.4321[\mathrm{M}-\mathrm{H}-132-132-162]^{-}$ & 0.171 & chikusetsusaponin $\mathrm{L}_{5}[39]$ \\
\hline 77 & 58.392 & 901.4614 & $769.4231[\mathrm{M}-\mathrm{H}-132]^{-}, 637.4352[\mathrm{M}-\mathrm{H}-132-132]^{-}, 475.4335[\mathrm{M}-\mathrm{H}-132-132-162]^{-}$ & 0.602 & notoginsenoside Rw1 [21] \\
\hline 78 & 58.569 & 783.4448 & $621.3941[\mathrm{M}-\mathrm{H}-146]^{-}, 475.3495[\mathrm{M}-\mathrm{H}-146-162]^{-}, 391.2896$ & 0.754 & ginsenoside $\mathrm{Rg}_{2}$ \\
\hline 79 & 59.146 & 1239.5732 & $\begin{array}{l}1107.6034[\mathrm{M}-\mathrm{H}-132]^{-}, 945.5396[\mathrm{M}-\mathrm{H}-132-162]^{-}, 783.5018[\mathrm{M}-\mathrm{H}-132-162-162]^{-}, \\
621.4381[\mathrm{M}-\mathrm{H}-132-162-162-162]^{-}, 459.5110[\mathrm{M}-\mathrm{H}-132-162-162-162-162]^{-}\end{array}$ & 2.087 & chikusetsusaponinVI [40] \\
\hline 80 & 60.406 & 637.3796 & $475.3437[\mathrm{M}-\mathrm{H}-162]^{-}, 391.2901$ & 0.103 & ginsenoside $F_{1}$ [27] \\
\hline 81 & 60.818 & 1269.5894 & $\begin{array}{l}1107.5013[\mathrm{M}-\mathrm{H}-162]^{-}, 945.4587[\mathrm{M}-\mathrm{H}-162-162]^{-}, 783.4201[\mathrm{M}-\mathrm{H}-162-162-162]^{-}, \\
621.3833[\mathrm{M}-\mathrm{H}-162-162-162-162]^{-}, 459.5120[\mathrm{M}-\mathrm{H}-162-162-162-162-162]^{-}\end{array}$ & 0.045 & ginsenoside $\mathrm{Ra}_{0}$ or quinquenoside $\mathrm{V}[19,21]$ \\
\hline 82 & 61.572 & 1239.5892 & $\begin{array}{l}1107.5974[\mathrm{M}-\mathrm{H}-132]^{-}, 945.5267[\mathrm{M}-\mathrm{H}-132-162]^{-}, 783.4965[\mathrm{M}-\mathrm{H}-132-162-162]^{-}, \\
621.4475[\mathrm{M}-\mathrm{H}-132-162-162-162]^{-}, 459.5121[\mathrm{M}-\mathrm{H}-132-162-162-162-162]^{-}\end{array}$ & 2.622 & notoginsenoside Fa \\
\hline 83 & 62.102 & 1105.5357 & $\begin{array}{l}943.5543[\mathrm{M}-\mathrm{H}-162]^{-}, 781.4996[\mathrm{M}-\mathrm{H}-162-162]^{-}, 619.3441[\mathrm{M}-\mathrm{H}-162-162-162]^{-}, \\
457.3654[\mathrm{M}-\mathrm{H}-162-162-162-162]^{-}\end{array}$ & 0.941 & PPT-B-S 1 (Glc-Glc-Glc-Glc) [38] \\
\hline 84 & 63.562 & 1105.5425 & $\begin{array}{l}943.5521[\mathrm{M}-\mathrm{H}-162]^{-}, 781.5010[\mathrm{M}-\mathrm{H}-162-162]^{-}, 619.3501[\mathrm{M}-\mathrm{H}-162-162-162]^{-}, \\
457.2601[\mathrm{M}-\mathrm{H}-162-162-162-162]^{-}\end{array}$ & 0.161 & isomer of PPT-B-S 1 (Glc-Glc-Glc-Glc) [38] \\
\hline 85 & 63.562 & 1239.2331 & $\begin{array}{l}1077.4231[\mathrm{M}-\mathrm{H}-162]^{-}, 915.4635[\mathrm{M}-\mathrm{H}-162-162]^{-}, 621.3862[\mathrm{M}-\mathrm{H}-162-162-294]^{-} \\
459.3452[\mathrm{M}-\mathrm{H}-162-162-294-162]^{-}\end{array}$ & 0.019 & ginsenoside $\mathrm{Ra}_{3}[39]$ \\
\hline 86 & 64.198 & 1107.5642 & $\begin{array}{l}945.4624[\mathrm{M}-\mathrm{H}-162]^{-}, 783.4239[\mathrm{M}-\mathrm{H}-162-162]^{-}, 621.3852[\mathrm{M}-\mathrm{H}-162-162-162]^{-} \\
459.3475[\mathrm{M}-\mathrm{H}-162-162-162-162]^{-}\end{array}$ & 32.21 & ginsenoside $\mathrm{Rb}_{1}$ \\
\hline 87 & 66.483 & 1107.5632 & $\begin{array}{l}945.4631[\mathrm{M}-\mathrm{H}-162]^{-}, 783.4197[\mathrm{M}-\mathrm{H}-162-162]^{-}, 621.3800[\mathrm{M}-\mathrm{H}-162-162-162]^{-}, \\
459.3452[\mathrm{M}-\mathrm{H}-162-162-162-162]^{-}\end{array}$ & 0.019 & isomer of ginsenoside $\mathrm{Rb}_{1}[12,40]$ \\
\hline 88 & 68.791 & 1077.5696 & $\begin{array}{l}945.4812[\mathrm{M}-\mathrm{H}-132]^{-}, 783.4405[\mathrm{M}-\mathrm{H}-132-162]^{-}, 621.4014[\mathrm{M}-\mathrm{H}-132-162-162]^{-}, \\
459.3421[\mathrm{M}-\mathrm{H}-132-162-162-162]^{-}\end{array}$ & 0.110 & notoginsenoside L $[12,27]$ \\
\hline 89 & 69.639 & 1077.5705 & $\begin{array}{l}945.4888[\mathrm{M}-\mathrm{H}-132]^{-}, 783.4465[\mathrm{M}-\mathrm{H}-132-162]^{-}, 621.4008[\mathrm{M}-\mathrm{H}-132-162-162]^{-}, \\
459.3544[\mathrm{M}-\mathrm{H}-132-162-162-162]^{-}\end{array}$ & 0.080 & isomer of notoginsenoside L $[12,27]$ \\
\hline 90 & 70.487 & 943.5121 & $781.4300[\mathrm{M}-\mathrm{H}-162]^{-}, 619.3881[\mathrm{M}-\mathrm{H}-162-162]^{-}, 457.3656[\mathrm{M}-\mathrm{H}-162-162-162]^{-}$ & 0.058 & PPT-B-S 1 (Glc-Glc-Glc) * \\
\hline 91 & 73.384 & 945.5495 & $783.4954[\mathrm{M}-\mathrm{H}-162]^{-}, 621.4422[\mathrm{M}-\mathrm{H}-162-162]^{-}, 459.3887[\mathrm{M}-\mathrm{H}-162-162-162]^{-}$ & 3.134 & ginsenoside Rd \\
\hline 92 & 77.824 & 945.5520 & $783.4964[\mathrm{M}-\mathrm{H}-162]^{-}, 621.4415[\mathrm{M}-\mathrm{H}-162-162]^{-}, 459.3887[\mathrm{M}-\mathrm{H}-162-162-162]^{-}$ & 0.370 & isomer of ginsenoside Rd $[9,12]$ \\
\hline 93 & 83.312 & 619.4279 & $457.3652[\mathrm{M}-\mathrm{H}-162]^{-}$ & 0.019 & ginsenoside $\mathrm{Rk}_{3}[41,42]$ \\
\hline 94 & 85.608 & 619.4255 & $457.3686[\mathrm{M}-\mathrm{H}-162]^{-}$ & 0.067 & ginsenoside $\mathrm{Rh}_{4}[41,42]$ \\
\hline
\end{tabular}



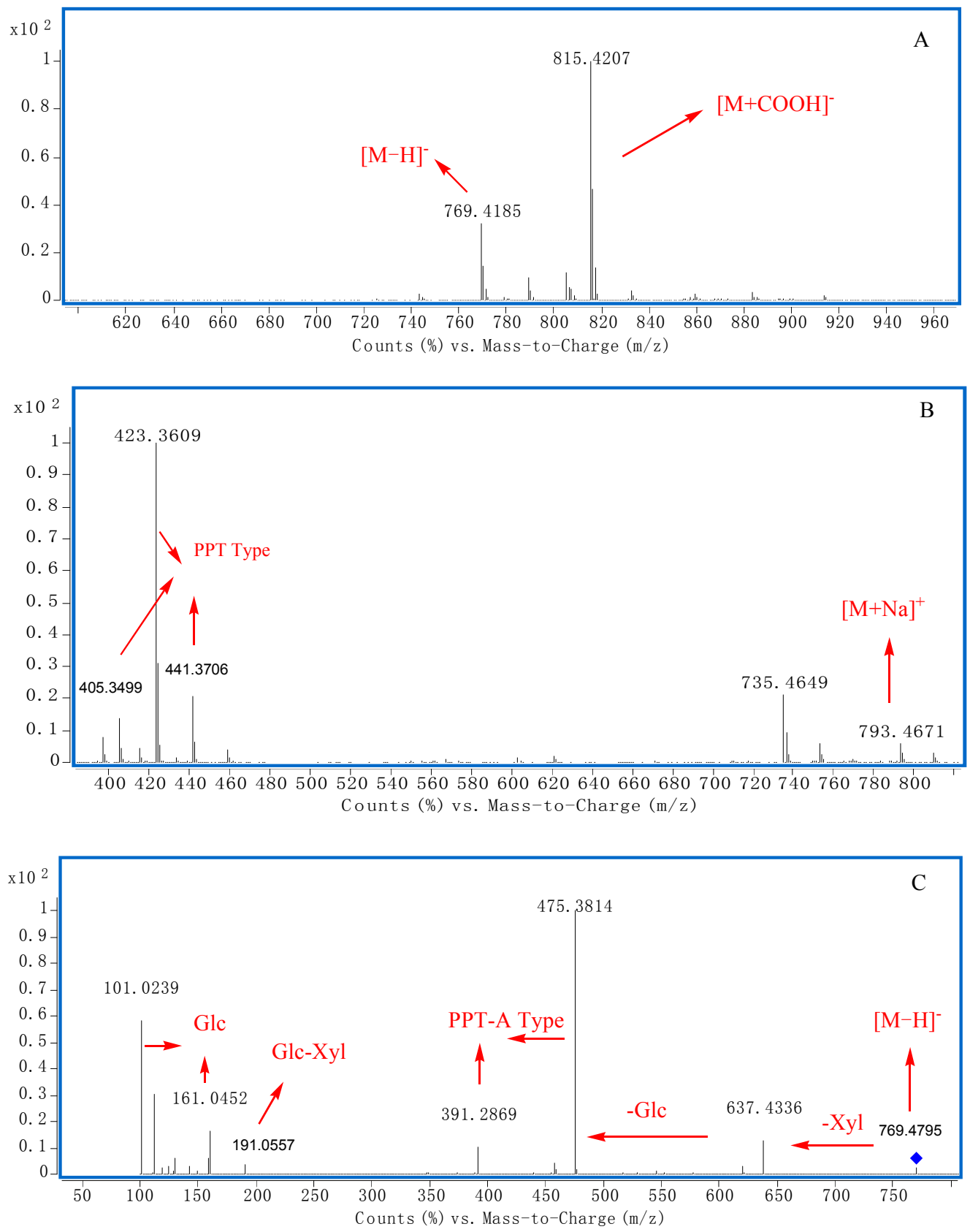

Figure 4. The typical TOF-MS spectra and fragmentation pathways of compound 72. (A) MS spectrum in negative mode; (B) MS spectrum in positive mode; (C) MS/MS spectrum in negative mode.

$[\mathrm{M}-\mathrm{H}]^{-}\left(\mathrm{m} / \mathrm{z}\right.$ 961.5409) and $[\mathrm{M}+\mathrm{COOH}]^{-}(\mathrm{m} / \mathrm{z}$ 1007.5460) peaks of compound 25 were observed in the negative MS spectrum, and the peak ratio of $[\mathrm{M}-\mathrm{H}]^{-}$to $[\mathrm{M}+\mathrm{COOH}]^{-}$was 1.5 , which indicated more than one sugar was located at the C-20 position. Diagnostic fragment ions at $m / z 799.4883[\mathrm{M}-\mathrm{H}-162]^{-}, 637.4389[\mathrm{M}-\mathrm{H}-162-162]^{-}, 475.3826[\mathrm{M}-\mathrm{H}-162-162-162]^{-}$, 391.2872 as well as $\mathrm{m} / \mathrm{z} 405.3973,423.4204,441.4223$ were observed in negative and positive mode, respectively. As a result, compound $\mathbf{2 5}$ was identified as notoginsenoside $R_{3}$, and compounds 37 and $\mathbf{7 1}$ were identified as isomers of $\mathbf{2 5}$ because similar fragment ions were observed in their spectra.

Compound 90 produced $[\mathrm{M}-\mathrm{H}]^{-}$ions at $m / z 943.5121$ and $[\mathrm{M}+\mathrm{COOH}]^{-}$ions at $m / z 989.5183$ in negative mode, while the peak ratio of $[\mathrm{M}-\mathrm{H}]^{-}$to $[\mathrm{M}+\mathrm{COOH}]^{-}$was 8 , which indicated there are two or more sugars at the C-20 position. Diagnostic ions of PPT type were observed at 
$m / z$ 405.3433, 423.3545, 441.3794, and diagnostic ions of PPT-A type were observed at $\mathrm{m} / \mathrm{z} 457.3656$. In the MS/MS spectrum, characteristic fragment ions at $m / z 781.4300[\mathrm{M}-\mathrm{H}-162]^{-}, 619.3881$ $[\mathrm{M}-\mathrm{H}-162-162]^{-}, 457.3656[\mathrm{M}-\mathrm{H}-162-162]^{-}$and Glc-Glc ions at $\mathrm{m} / \mathrm{z} 221.0662$ were obtained. Based on the MS cleavage rules (Tables 1 and 2), literature data and a SciFinder database search, compound 90 was tentatively identified as PPT-B-S 1 (Glc-Glc-Glc).

The molecular weight of compound 83 could be supposed to 1106 through negative ions at $m / z 1105.5357[\mathrm{M}-\mathrm{H}]^{---}$and $1151.5401[\mathrm{M}+\mathrm{COOH}]^{-}$(Tables 1 and 2). There must be less than one sugar at $\mathrm{C}-20$ position according to the peak ratio of $[\mathrm{M}-\mathrm{H}]^{-}$to $[\mathrm{M}+\mathrm{COOH}]^{-}(0.1)$. Furthermore, diagnostic ions of PPT type were observed at $m / z 405.3459,423.3565,441.3661$. In the MS/MS spectrum, fragment ions at $m / z 943.5543[\mathrm{M}-\mathrm{H}-162]^{-}, 781.4996[\mathrm{M}-\mathrm{H}-162-162]^{-}$, $619.3441[\mathrm{M}-\mathrm{H}-162-162-162]^{-}, 457.3654[\mathrm{M}-\mathrm{H}-162-162-162-162]^{-}$could be deduced to represent neutral losses of four glucose moieties successively from the precursor ions. Glc-Glc diagnostic ions at $m / z 221.0664$ could be detected. Characteristic ions at $m / z 457$ indicated the aglycone type of 83 should be PPT-B (Figure 1). According to the above elucidation and literature

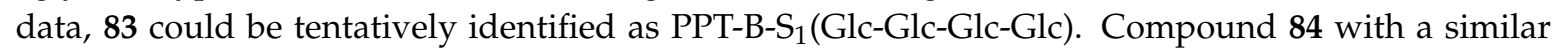
fragmentation behavior could be tentatively identified as an isomer of $\mathbf{8 3}$ (Tables 1 and 2).

Compound 12 produced $[\mathrm{M}-\mathrm{H}]^{-}$ions at $m / z 817.4872$ and $[\mathrm{M}+\mathrm{COOH}]^{-}$ions at $m / z 863.4982$ in the negative spectrum, while the peak ratio of $[\mathrm{M}-\mathrm{H}]^{-}$to $[\mathrm{M}+\mathrm{COOH}]^{-}$was 0.8 , which indicated there is more than one sugar at C-20. In MS/MS mode, characteristic fragment ions at $\mathrm{m} / \mathrm{z} 655.4462$ $[\mathrm{M}-\mathrm{H}-162]^{-}, 493.3934[\mathrm{M}-\mathrm{H}-162-162]^{-}$and 323. 2112 [Glc-Glc] $^{-}$were obtained. In positive mode, it produced ions at $m / z 423.3736$ and 441.3848 , which could be used to identify it as a PPT type, and in MS/MS mode, PPT-C type characteristic fragment ions at $\mathrm{m} / \mathrm{z} 493.3934$ was observed. According to retention time and MS fragment rules, compound $\mathbf{1 2}$ was tentatively identified as PPT-C-S 1 (Glc-Glc)-S 2 (H).

By utilizing MS cleavage rules, as well as comparison of the retention time to references, compounds 27, 29, 31, 33, 40, 41, 47, 55, 66, 73, 78, 93 and 94 were unambiguously identified as notoginsenoside $\mathrm{N}$, notoginsenoside $\mathrm{R}_{6}, 20$ - $\mathrm{O}$-glucoginsenoside $\mathrm{Rf}$, notoginsenoside $\mathrm{R}_{1}$, ginsenoside $\mathrm{Rg}_{1}$, ginsenoside $\mathrm{Re}$, pseudoginsenoside $\mathrm{Rt}_{3}$, sanchirhinoside $\mathrm{A}_{3}$, sanchirhinoside $\mathrm{B}$, notoginsenoside $\mathrm{U}$, ginsenoside $\mathrm{Rg}_{2}$, ginsenoside $\mathrm{Rk}_{3}$, and ginsenoside $\mathrm{Rh}_{4}$, respectively (Tables 1 and 2).

Similarly, by utilizing MS cleavage rules and literature data, another 27 PPT type saponins were identified or tentatively identified as ginsenoside $\operatorname{Rf}(\mathbf{1 3})$, yesanchinoside $\mathrm{E}$ (28), an isomer of yesanchinoside $\mathrm{E}$ (30), an isomer of 20-O-glucoginsenoside $\mathrm{Rf}$ (32), notoginsenoside $\mathrm{M}$ or $\mathrm{N}$ (34), an isomer of notoginsenoside $\mathrm{M}$ or $\mathrm{N}$ (35), gypenoside LXIV (36),

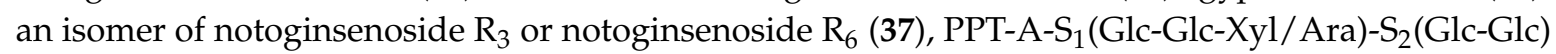
(42), quinquenoside $\mathrm{L}_{16}$ (43), PPT-C- $\mathrm{S}_{1}\left(\right.$ Glc)-S $\mathrm{S}_{2}\left(\right.$ Glc-Glc) (44), PPT-A-S $\mathrm{S}_{1}\left(\right.$ Glc-Glc)-S $\mathrm{S}_{2}$ (Glc-Glc) (45), chikusetsusaponin $\mathrm{L}_{5}$ (46), an isomer of chikusetsusaponin $\mathrm{L}_{5}$ (48), an isomer of notoginsenoside $R_{2}$ (49), an isomer of notoginsenoside $R_{2}$ (52), an isomer of 20-O-glucoginsenoside $\mathrm{Rf}$ (53), an isomer of PPT-A-S $S_{1}$ (Glc-Glc)-S $S_{2}$ (Glc-Glc) (54), PPT-A-S $S_{1}\left(\right.$ Rha-Xyl)-S $S_{2}($ Glc) (56), an isomer of PPT-A-S ${ }_{1}\left(\right.$ Glc-Glc)-S ${ }_{2}$ (Glc-Glc) (57), an isomer of PPT-A-S (Glc-Glc)-S $_{2}$ (Glc-Glc) (58), an isomer of

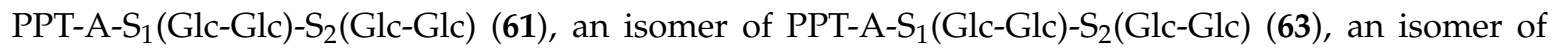
20-O-glucoginsenoside $\mathrm{Rf}(67)$, an isomer of notoginsenoside $\mathrm{R}_{3}$ or an isomer of notoginsenoside $\mathrm{R}_{6}$ (68), ginsenoside $\mathrm{Rg}_{1}(69)$, an isomer of 20-O-glucoginsenoside $\mathrm{Rf}(70)$, an isomer of notoginsenoside $R_{3}$ or an isomer of notoginsenoside $R_{6}(71)$, an isomer of notoginsenoside $R_{2}(72)$ and ginsenoside $F_{1}$ (78), respectively (Tables 1 and 2).

\subsubsection{Identification of OCO Type Saponins}

The molecular weight of compound 9 could be supposed to 816 through negative ions at $m / z 861.4817[\mathrm{M}+\mathrm{COOH}]^{-}$and $815.4669[\mathrm{M}-\mathrm{H}]^{-}$(Figure 5A,B). There must be less than one sugar moiety at the $\mathrm{C}-20$ position according to the peak ratio of $[\mathrm{M}-\mathrm{H}]^{-}$to $[\mathrm{M}+\mathrm{COOH}]^{-}(0.02)$. Furthermore, diagnostic ions of OCO type was observed at $m / z$ 421, 439, 457 (Figures 1 and 5B). 
In the MS/MS spectrum of $\left[\mathrm{M}+\mathrm{HCOO}^{-}\right.$(Figure 5C), fragment ions at $\mathrm{m} / \mathrm{z} 653.4263,491.3777$ could be deduced to correspond to neutral losses of two glucoses successively from the precursor ions. Diagnostic ions at $m / z 323$ could be used for confirming a Glc-Glc chain located at the C-6 position. Characteristic ions at $\mathrm{m} / z 415$ and 491 indicated the aglycone type of 9 should be OCO-C (Figures 1 and $5 \mathrm{C}$ ). According to the above elucidation and literature data, 9 could be tentatively identified as majonoside $\mathrm{R}_{1}[26,43]$. Compounds 10, 11, 16, 17 had similar fragmentation behaviors at $m / z 653.4263$ [M $-\mathrm{H}-162]^{-}, 491.3777$ [M $\left.-\mathrm{H}-162-162\right]^{-}, 415.3253$. Glc-Glc diagnostic ions at $m / z 323$ and diagnostic ions of OCO type was also observed at $m / z 421,439,457$, so compounds 10, $\mathbf{1 1}, \mathbf{1 6}, 17$ were tentatively identified as isomers of majonoside $R_{1}$ since similar characteristic fragment ions were obtained (Tables 1 and 2).
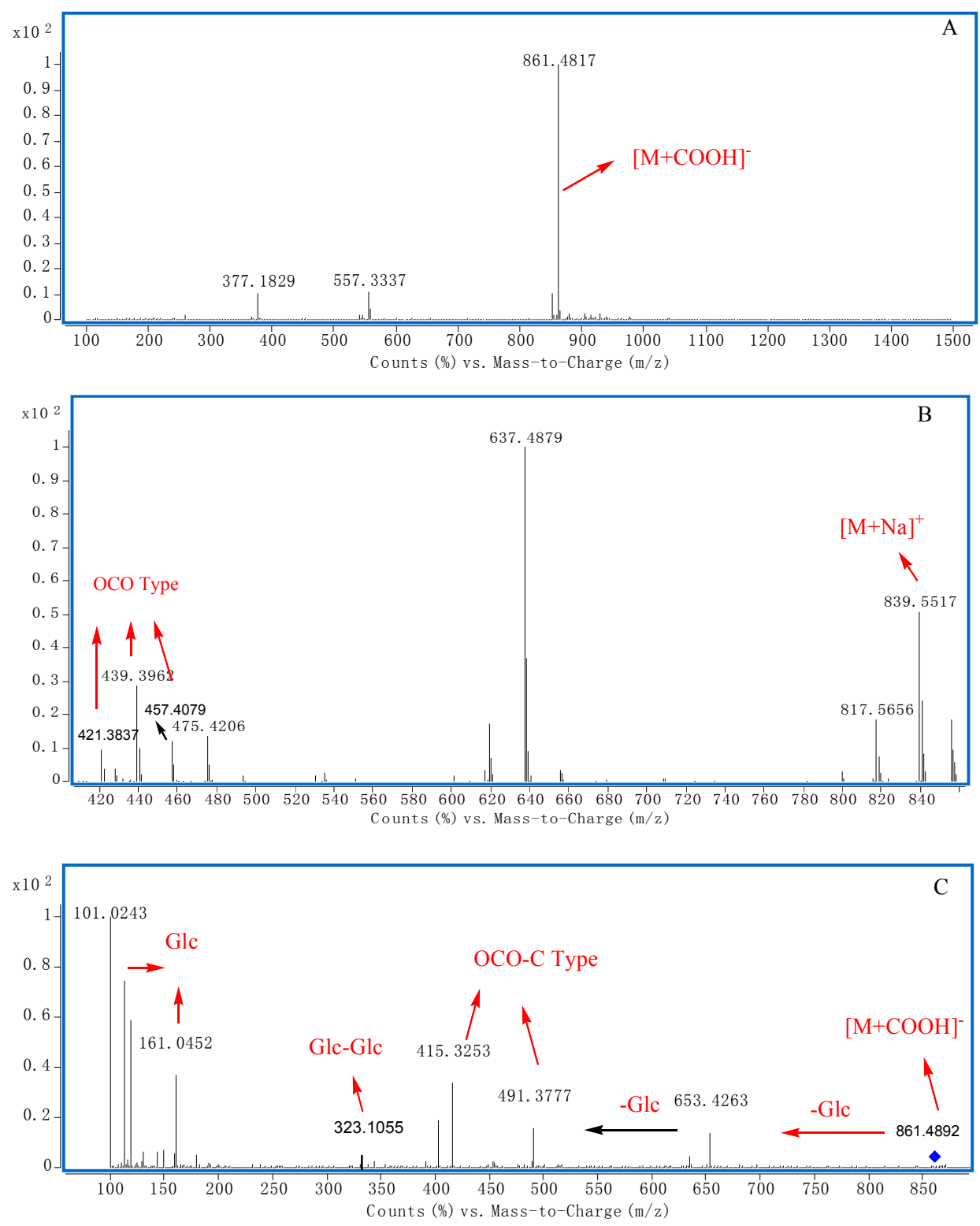

Figure 5. The typical TOF-MS spectra and fragmentation pathways of compound 9. (A) MS spectrum in negative mode; (B) MS spectrum in positive mode; (C) MS/MS spectrum in negative mode.

$[\mathrm{M}-\mathrm{H}]^{-}\left(\mathrm{m} / \mathrm{z}\right.$ 947.4713) and $[\mathrm{M}+\mathrm{COOH}]^{-}(\mathrm{m} / \mathrm{z}$ 993.4633) of compound 64 were observed in the negative MS spectrum, and the peak ratio of $[\mathrm{M}-\mathrm{H}]^{-}$to $[\mathrm{M}+\mathrm{COOH}]^{-}$was 0.25 . Diagnostic fragment ions at $\mathrm{m} / \mathrm{z} 815.3567[\mathrm{M}-\mathrm{H}-132]^{-}, 653.3778[\mathrm{M}-\mathrm{H}-132-162]^{-}$, 
491.3608 [M - H - $132-162-162]^{-}, 391.8554$, as well as $\mathrm{m} / z$ 421.3447, 439.3612, 457.3701 were observed in negative and positive mode, respectively. As a result, 64 was identified as OCO-B-S ${ }_{1}\left(\right.$ Glc-Glc-Xyl/Ara)-S $\mathrm{S}_{2}(\mathrm{OH})$.

The molecular weight of compound 1 could be deduced as 966 according to quasi-molecular ions at $m / z 965.5198[\mathrm{M}-\mathrm{H}]^{-}$and $1011.5278[\mathrm{M}+\mathrm{COOH}]^{-}$. The peak ratio of $[\mathrm{M}-\mathrm{H}]^{-}$to $[\mathrm{M}+\mathrm{COOH}]^{-}$was about 0.1 , which indicated that there was one or less sugar at $\mathrm{C}-20$. The aglycone type could be identified as OCO type through diagnostic ions at $m / z 439,457$. In the MS/MS spectrum of $[\mathrm{M}-\mathrm{H}]^{-}$, fragment ions at $m / z 785.9465[\mathrm{M}-\mathrm{H}-180]^{-}, 653.3969[\mathrm{M}-\mathrm{H}-180-132]^{-}, 491.3843$ $[\mathrm{M}-\mathrm{H}-180-132-162]^{-}, 415.3350$ and fragment ions at $m / z 191$ indicated there was a Glc-Xyl/Ara chain in compound 1. Characteristic ions at $\mathrm{m} / \mathrm{z} 415$ and 491 indicated the aglycone would be OCO-A type. According to the peak ratio rule of $[\mathrm{M}-\mathrm{H}]^{-}$to $[\mathrm{M}+\mathrm{COOH}]^{-}$and the structural features of OCO-A, we could deduce that the Glc-Xyl chain was linked at the C-6 position of the aglycone. As a result, compound 1 was tentatively identified as OCO-A-S $\left(\right.$ Glc)-S $S_{2}$ (Glc-Xyl/Ara), compound $\mathbf{2}$ was identified as an isomer of $\mathbf{1}$ because a similar MS cleavage pathway was observed.

Compound 18 could be supposed to have a molecular weight of 814 through negative ions at $\mathrm{m} / \mathrm{z}$ $813.4677[\mathrm{M}-\mathrm{H}]^{-}$and $859.4739[\mathrm{M}+\mathrm{COOH}]^{-}$(Figure 6). There must be less than one sugar at the C-20 position according to the peak ratio $(0.1)$ of $[\mathrm{M}-\mathrm{H}]^{-}$to $[\mathrm{M}+\mathrm{COOH}]^{-}$. Furthermore, diagnostic ions of OCO type was observed at $m / z 421,439,457$ (Figure 1). In the MS/MS spectrum, fragment ions at $m / z 651.4111[\mathrm{M}-\mathrm{H}-162]^{-}$and $489.3579[\mathrm{M}-\mathrm{H}-162-162]^{-}$could be deduced as successive neutral losses of two glucose molecules (Figure 6). Characteristic ions at $m / z 489$ indicated the aglycone type should be OCO-E. According to the above elucidation and literature data, $\mathbf{1 8}$ could be tentatively identified as OCO-E-S $\mathrm{S}_{1}(\mathrm{Glc}-\mathrm{Glc})$. Compound $\mathbf{2 1}$ with similar fragmentation behaviors at $m / z 651.4113[\mathrm{M}-\mathrm{H}-162]^{-}, 489.3635[\mathrm{M}-\mathrm{H}-162-162]^{-}$, was tentatively identified as an isomer of OCO-E-S $($ Glc-Glc).

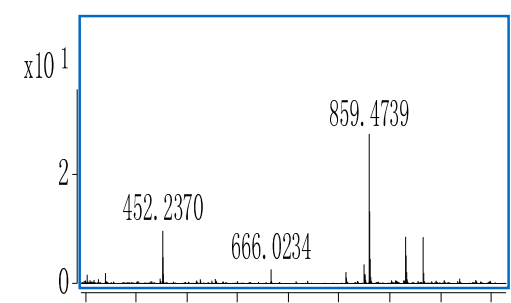

4005006007008009001000

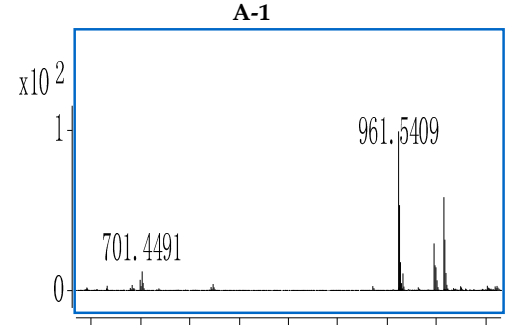

$\begin{array}{lllllll}700 & 750 & 800 & 850 & 900 & 950 & 1000\end{array}$

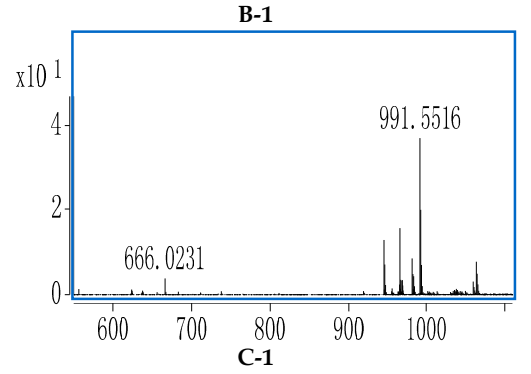

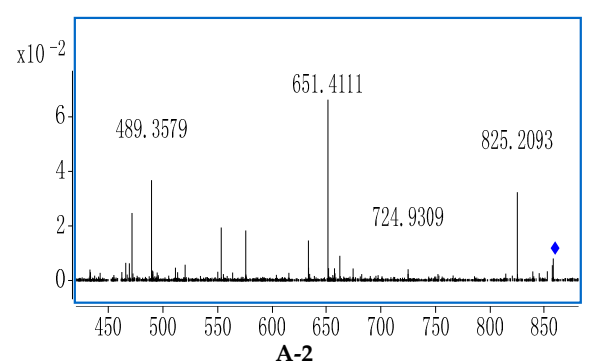
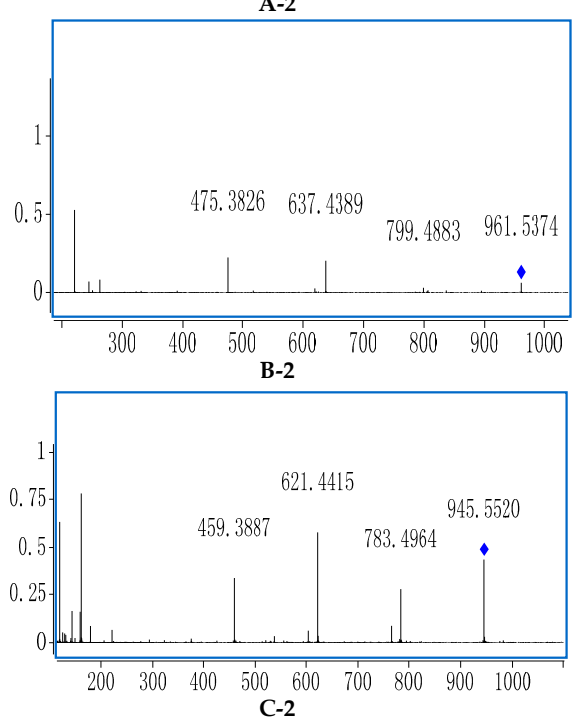

Figure 6. The typical TOF-MS spectra of compounds 18 (A); 25 (B); 92 (C) in negative mode (1: MS spectra; 2: MS/MS spectra). 
In the negative MS spectrum of compound 20, $[\mathrm{M}-\mathrm{H}]^{-}$ions at $\mathrm{m} / z 959.5194$ and $[\mathrm{M}+\mathrm{COOH}]^{-}$ at 1005.5220 as well as their peak ratio (0.25) were observed. In the MS/MS spectrum, characteristic fragment ions at $m / z 797.4207[\mathrm{M}-\mathrm{H}-162]^{-}, 635.3567[\mathrm{M}-\mathrm{H}-162-162]^{-}, 473.3460$ [M $-\mathrm{H}-162-162-162]^{-}$and 221.0431 [Glc-Glc] $^{-}$were also observed. In the positive spectrum it produced ions at $m / z 421,439,457$ which could be used to identify an OCO type compound. In MS/MS mode, OCO-D type fragment ions at $\mathrm{m} / \mathrm{z} 473$ could be obtained. According to the MS fragment rules, 20 was tentatively identified as OCO-D-S ${ }_{1}$ (Glc-Glc-Glc). Similar diagnostic fragment ions were observed in $\mathbf{2 2}$ (Tables 1 and 2).

Similarly, with the help of MS cleavage rules and literature values, compounds 7, 14, 15, 19, 51 were tentatively identified as vinaginsenoside $R_{5}$ or yesanchinoside $C$, OCO-B-S $\mathrm{S}_{1}$ (Glc-Rha-Glc)-S $\mathrm{S}_{2}\left(\mathrm{CH}_{3}\right)$, an isomer of OCO-B-S $\mathrm{S}_{1}$ (Glc-Rha-Glc)- $\mathrm{S}_{2}\left(\mathrm{CH}_{3}\right)$, an isomer of OCO-B-S ${ }_{1}\left(\right.$ Glc-Rha-Glc)- $\mathrm{S}_{2}\left(\mathrm{CH}_{3}\right)$ and OCO-B-S ${ }_{1}(\mathrm{Xyl}-\mathrm{Glc}-\mathrm{Glc}-\mathrm{Glc})-\mathrm{S}_{2}(\mathrm{OH})$ (Tables 1 and 2).

\section{Experimental Section}

\subsection{Reagents and Materials}

HPLC grade acetonitrile (ACN) and formic acid were purchased from Merck Technologies Inc. (Darmstadt, Germany), and Tedia Company Inc. (Fairfield, OH, USA). Deionized water was obtained from a Millipore Milli-Q water system (Bedford, MA, USA). All other reagents were of analytical purity. ZSYXST sample was obtained from Guangxi Wuzhou Pharmaceutical (Group) Co., Ltd. (Wuzhou, China). Twenty-one reference compounds: sanchirhinoside $A_{6}(23)$, notoginsenoside $R_{3}$ (25), notoginsenoside $N(27)$, notoginsenoside $R_{6}$ (29), 20(S)-20-O-glucoginsenoside $R f(31)$, notoginsenoside $R_{1}$ (33), notoginsenoside $G$ (39), ginsenoside $\operatorname{Rg}_{1}$ (40), ginsenoside $\operatorname{Re}(41)$, pseudoginsenoside $\mathrm{Rt}_{3}(47)$, sanchirhinoside $\mathrm{A}_{3}$ (55), vina-ginsenoside $\mathrm{R}_{4}(65)$, sanchirhinoside $\mathrm{B}$ (66), notoginsenoside $R_{2}(72)$, notoginsenoside $U(73)$, ginsenoside $\operatorname{Rg}_{2}(78)$, notoginsenoside $F a(82)$, ginsenoside $\mathrm{Ra}_{3}(85)$, ginsenoside $\mathrm{Rb}_{1}(\mathbf{8 6})$, ginsenoside $\mathrm{Rd}(\mathbf{9 1})$, ginsenoside $\mathrm{Rk}_{3}(\mathbf{9 3})$, and ginsenoside $\mathrm{Rh}_{4}(94)$ were isolated from ZSYXST by the authors. Their structures were elucidated by $1 \mathrm{D}$ and $2 \mathrm{D}$ NMR spectra $[28,44]$.

\subsection{Sample Preparation and PHPLC Chromatography Conditions}

ZSYXST (10 mg)was dissolved in 19\% acetonitrile $(1 \mathrm{~mL})$ to obtain sample 1, which was then centrifuged for $10 \mathrm{~min}$ at 14,000 rpm, and the supernatant of sample 1 was applied to a Shimadzu LC-8A PHPLC system (Shimadzu, Kyoto, Japan), equipped with a binary pump, an UV detector and a fraction collector(FRC-10A). Chromatographic separation was achieved on a Cosmosil-5 $\mathrm{C}_{18}$ column $(20 \times 250 \mathrm{~mm}, 5 \mu \mathrm{m})$, (NACALAI, Kyoto, Japan). The mobile phase consisted of water (A) and ACN (B), using $19 \%-20 \%$ B at $0-25 \mathrm{~min}, 20 \%-30 \%$ B at $25-35 \mathrm{~min}, 30 \%-35 \%$ B at $35-45 \mathrm{~min}, 35 \%-45 \%$ B at $45-70 \mathrm{~min}, 45 \%-90 \%$ B at $70-75 \mathrm{~min}, 90 \% \mathrm{~B}$ at $75-76 \mathrm{~min}, 90 \%-19 \% \mathrm{~B}$ at $76-80 \mathrm{~min}$, the flow rate was $8 \mathrm{~mL} / \mathrm{min}$.

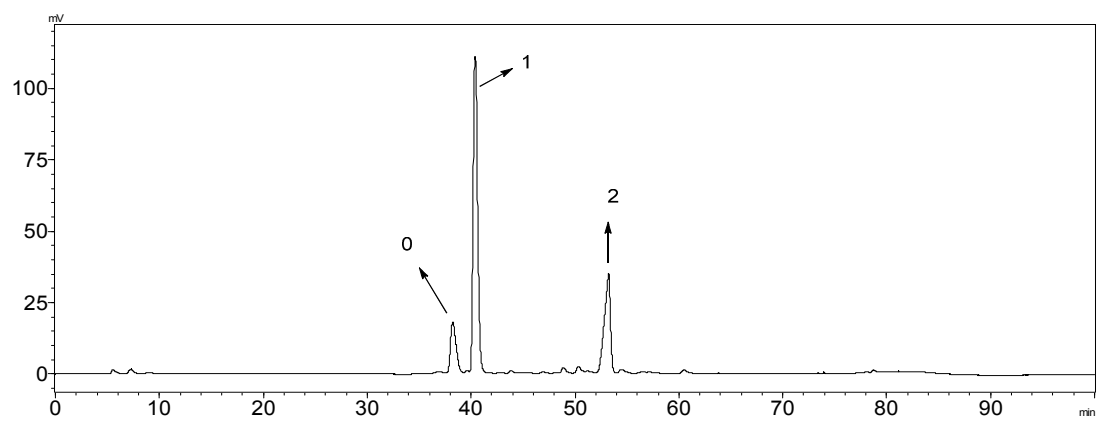

Figure 7. The PHPLC chromatogram of sample 1 ( 0 : Notoginsenoside $\mathrm{R}_{1} ; 1$ : Ginsenoside $\operatorname{Rg} 1$ and $\operatorname{Re}$; 2: Ginsenoside $\mathrm{Rb} 1)$. 
Utilizing an online collector simulation system, the major peaks 0-2 of ZSYXST were easily removed through the control of the collection parameters, set at level of $500 \mu \mathrm{V}$, peak slope of $1000 \mu \mathrm{V} / \mathrm{s}$ and the delay volume of $200 \mu \mathrm{L}$. Finally, sample $2(1.3 \mathrm{mg})$ without major components was obtained, and the peaks of lower content ingredients became more obvious finally (Figures 7 and 8 ).

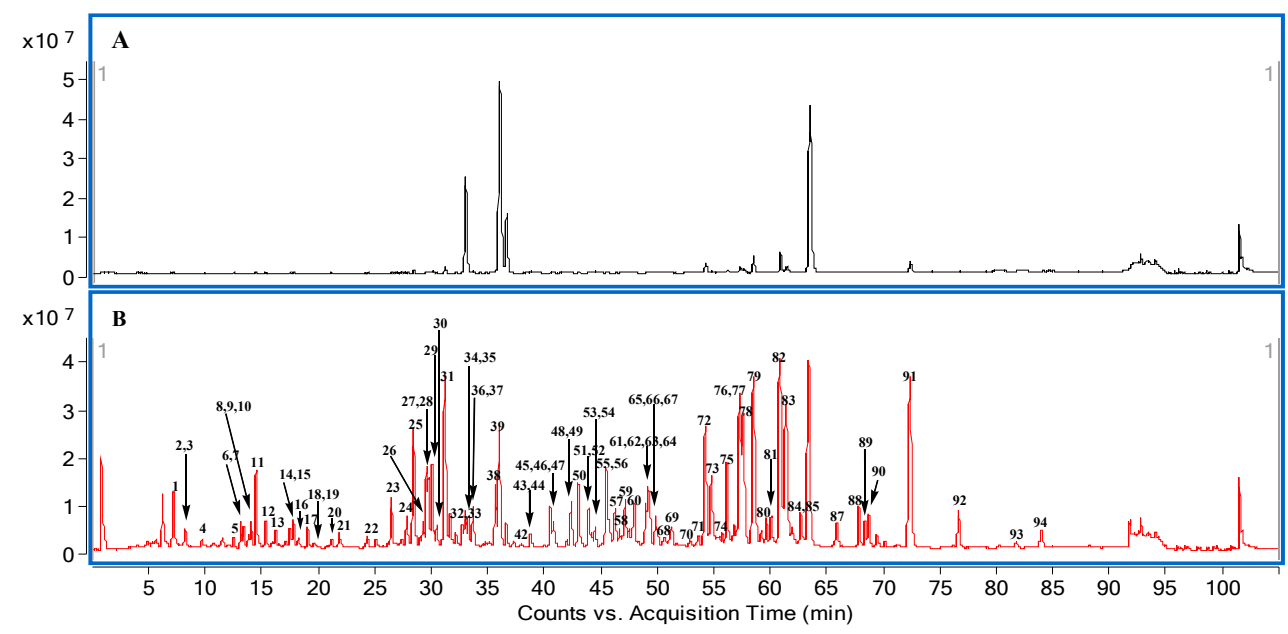

Figure 8. The total ion chromatograms of UHPLC-Q-TOF/MS in negative mode.

\subsection{UHPLC-Q-TOF/MS Conditions}

Sample $2(1 \mathrm{mg} / \mathrm{mL})$ and ZSYXST sample $(1 \mathrm{mg} / \mathrm{mL})$ were analyzed on an Agilent 1290 Ultra high performance liquid chromatography system (Agilent, Palo alto, CA, USA), equipped with a $\mathrm{T}_{3}$ column $\left(2.1 \times 100 \mathrm{~mm}, 1.8 \mu \mathrm{m}\right.$, Waters, Milford, MA, USA) under $40^{\circ} \mathrm{C}$. The mobile phase consisted of $0.1 \%$ formic acid water (A) and ACN (B), using 10\% B at $0-10 \mathrm{~min}, 10-40 \% \mathrm{~B}$ at $10-90 \mathrm{~min}, 40 \%-100 \% \mathrm{~B}$ at $90-91 \mathrm{~min}, 100 \% \mathrm{~B}$ at $91-100 \mathrm{~min}, 100 \%-10 \% \mathrm{~B}$ at $100-100.1 \mathrm{~min}, 10 \% \mathrm{~B}$ at 100.1-105 $\mathrm{min}$. The flow rate was $0.3 \mathrm{~mL} / \mathrm{min}$, and the sample volume injected was $2 \mu \mathrm{L}$.

The Q-TOF/MS analysis was performed on an Agilent 6520 Accurate-Mass Q-TOF/MS system. The conditions of the ESI source were: drying gas $\left(\mathrm{N}_{2}\right)$ flow rate, $8.0 \mathrm{~L} / \mathrm{min}$; drying gas temperature, $350{ }^{\circ} \mathrm{C}$; nebulizer, 30 psig; capillary voltage (Vcap), 3500V. Cone voltage was $120 \mathrm{~V}$ and $175 \mathrm{~V}$ in the positive and negative mode, respectively. Collision energy (CE) was dynamically adjusted from 45 to $70 \mathrm{~V}$ according to the $\mathrm{m} / \mathrm{z}$ of precursor ions in the negative MS/MS mode. The mass analyzer scanned over $m / z$ 100-2000. All the data were recorded and processed by Agilent Mass Hunter Workstation software (Version B.02.00).

\section{Conclusions}

In this work, a novel UHPLC-Q-TOF-MS combined with PHPLC method was established. The sensitivity of some minor components in ZSYXST could be enhanced significantly by this method. Combining the characteristic ions in positive and negative mode, the types of aglycone, saccharide, as well as the linkage positions of the saccharide chains of saponins were quickly determined. As a result, based on the exact mass, fragmentation behaviors, retention times and literature, 94 saponins, including 20 pairs of isomers, which could represent over $98 \%$ of the components in ZSYXST were identified or tentatively identified and ten of these saponins were identified as new compounds. This method could provide a powerful platform for profiling the compounds in ZSYXST and also could be useful for identification of saponins of P. notoginseng and P. ginseng.

Acknowledgments: This study was supported by Important Drug Development Fund, The National Science Fund for Distinguished Young Scholars (81125024), Ministry of Science and Technology of China (No. 2012ZX09101201-002, 2012ZX09304007), Program for Innovative Research Team in Universities of Tianjin (TD12-5033) and Program for New Century Excellent Talents in University (NCET-12-1069). 
Author Contributions: Wang L-L and Han L-F made the same contribution on this research. Linlin Wang, Heshui Yu, and Mangmang Sang performed the experiment; Lifeng Han wrote the manuscript; Erwei Liu, Yi Zhang, Shiming Fang, and Tao Wang perfected language; Xiumei Gao designed the research. All authors read and approved the final version.

Conflicts of Interest: The authors declare no conflict of interest.

\section{References}

1. Liu, H.; Liang, J.P.; Li, P.B.; Peng, W.; Peng, Y.Y.; Zhang, G.M.; Xie, C.S.; Long, C.F.; Su, W.W. Core bioactive components promoting blood circulation in the traditional Chinese medicine compound Xueshuantong capsule $(\mathrm{CXC})$ based on the relevance analysis between chemical HPLC fingerprint and in vivo biological effects. PLoS ONE 2014, 9, e112675.

2. Gui, Q.F.; Yang, Y.M.; Ying, S.H.; Zhang, M.M. Xueshuantong improves cerebral blood perfusion in elderly patients with lacunar infarction. Neural Regen. Res. 2013, 8, 792-801.

3. Gao, L.; Zhao, H.; Liu, Q.; Song, J.; Xu, C.; Liu, P.; Gong, W.; Wang, R.; Liu, K.J.; Luo, Y. Improvement of hematoma absorption and neurological function in patients with acute intracerebral hemorrhage treated with Xueshuantong. J. Neurol. Sci. 2013, 325, 190. [CrossRef]

4. Yuan, Y.Z.; Yuan, F.; Xu, Q.Y.; Yu, J.; Li, L.; Zhang, J.L. Effect of Fufang Xueshuantong Capsule on a rat model of retinal vein occlusion. Chin. J. Integr. Med. 2011, 17, 296-301.

5. Zhang, Q.; Xiao, X.; Li, M.; Li, W.; Yu, M.; Zhang, H.; Sun, X.; Mao, L.; Xiang, H. Attenuating effect of Fufang Xueshuantong Capsule on kidney function in diabetic nephropathy model. J. Nat. Med. 2013, 67, 86-97. [PubMed]

6. Duan, H.; Huang, J.; Li, W.; Tang, M. Protective effects of fufang xueshuantong on diabetic retinopathy in rats. Evid. Based. Complement. Altern. Med. 2013, 408268. [CrossRef] [PubMed]

7. Chen, M.; Yang, W.Z.; Wu, W.Y.; Guo, D.A. Chemical Analysis of Xueshuantong Lyophilized Powder by LC-MS Profiling. Chin. Herb. Med. 2015, 7, 54-61.

8. Plumb, R.; CastrO-Perez, J.; Granger, J.; Beattie, I.; Joncour, K.; Wright, A. Ultra-performance liquid chromatography coupled to quadrupole-Orthogonal time-of-flight mass spectrometry. Rapid Commun. Mass Spectrom. 2004, 18, 2331-2337. [CrossRef] [PubMed]

9. Han, L.; Pan, G.; Wang, Y.; Song, X.; Gao, X.; Ma, B.; Kang, L. Rapid profiling and identification of triterpenoid saponins in crude extracts from Albizia julibrissin Durazz. by ultra high-performance liquid chromatography coupled with electrospray ionization quadrupole time-of-flight tandem mass spectrometry. J. Pharm. Biomed. Anal. 2011, 55, 996-1009. [CrossRef] [PubMed]

10. Zhu, J.; Guo, X.; Fu, S.; Zhang, X.; Liang, X. Characterization of steroidal saponins in crude extracts from Dioscorea zingiberensis C. H. Wright by ultra-performance liquid chromatography/electrospray ionization quadrupole time-of-flight tandem mass spectrometry. J. Pharm. Biomed. Anal. 2010, 53, 462-474. [CrossRef] [PubMed]

11. Lai, C.; Tan, T.; Zeng, S.; Qi, L.W.; Liu, X.; Dong, X.; Li, P.; Liu, E.H. An integrated high resolution mass spectrometric data acquisition method for rapid screening of saponins in Panax notoginseng (Sanqi). J. Pharm. Biomed. Anal. 2015, 109, 184-191. [CrossRef] [PubMed]

12. Qi, L.W.; Wang, H.Y.; Zhang, H.; Wang, C.Z.; Li, P.; Yuan, C.S. Diagnostic ion filtering to characterize ginseng saponins by rapid liquid chromatography with time-of-flight mass spectrometry. J. Chromatogr. A 2012, 1230, 93-99. [CrossRef] [PubMed]

13. Sun, S.; Xie, Z.S.; Liu, E.H.; Yan, Y.T.; Xu, X.J.; Li, P. Chemical profiling of Jinqi Jiangtang tablets by HPLC-ESI-Q-TOF/MS. Chin. J. Nat. Med. 2014, 12, 229-240. [CrossRef]

14. Li, L.; Luo, G.A.; Liang, Q.L.; Hu, P.; Wang, Y.M. Rapid qualitative and quantitative analyses of Asian ginseng in adulterated American ginseng preparations by UPLC/Q-TOF-MS. J. Pharm. Biomed. Anal. 2010, 52, 66-72. [CrossRef] [PubMed]

15. Mao, Q.; Yang, J.; Cui, X.M.; Li, J.J.; Qi, Y.T.; Zhang, P.H.; Wang, Q. Target separation of a new anti-tumor saponin and metabolic profiling of leaves of Panax notoginseng by liquid chromatography with eletrospray ionization quadrupole time-of-flight mass spectrometry. J. Pharm. Biomed. Anal. 2012, 59, 67-77. [CrossRef] [PubMed] 
16. Guan, J.; Lai, C.M.; Li, S.P. A rapid method for the simultaneous determination of 11 saponins in Panax notoginseng using ultra performance liquid chromatography. J. Pharm. Biomed. Anal. 2007, 44, 996-1000. [CrossRef] [PubMed]

17. Liu, J.H.; Wang, X.; Cai, S.Q.; Komatsu, K.; Namba, T. Analysis of the Constituents in the Chinese Drug Notoginseng by Liquid Chromatography-Electrospray Mass Spectrometry. J. Chin. Pharm. Sci. 2004, 13, 225-235.

18. Yang, W.Y.; Bo, T.; Ji, S.; Qiao, X.; Guo, D.A.; Ye, M. Rapid chemical profiling of saponins in the flower buds of Panax notoginseng by integrating MCI gel column chromatography and liquid chromatography/mass spectrometry analysis. Food Chem. 2013, 139, 762-769. [CrossRef] [PubMed]

19. Christensen, L.P. Ginsenosides: Chemistry, biosynthesis, analysis, and potential health effects. Adv. Food Nutr. Res. 2009, 55, 1-99. [PubMed]

20. Qi, L.W.; Wang, C.Z.; Yuan, C.S. American ginseng: Potential structure-function relationship in cancer chemoprevention. Biochem. Pharmacol. 2010, 80, 947-954. [CrossRef] [PubMed]

21. Yang, W.Z.; Ye, M.; Qiao, X.; Liu, C.F.; Miao, W.J.; Bo, T.; Tao, H.Y.; Guo, D.A. A strategy for efficient discovery of new natural compounds by integrating orthogonal column chromatography and liquid chromatography/mass spectrometry analysis: Its application in Panax ginseng, Panax quinquefolium and Panax notoginseng to characterize 437 potential new ginsenosides. Anal. Chim. Acta 2012, 739, 56-66. [PubMed]

22. Wu, W.; Sun, L.; Zhang, Z.; Guo, Y.; Liu, S. Profiling and multivariate statistical analysis of Panax ginseng based on ultra-high-performance liquid chromatography coupled with quadrupole-time-of-flight mass spectrometry. J. Pharm. Biomed. Anal. 2015, 107, 141-150. [CrossRef] [PubMed]

23. Li, S.; Tang, Y.; Liu, C.; Li, J.; Guo, L.; Zhang, Y. Development of a method to screen and isolate potential xanthine oxidase inhibitors from Panax japlcus var via ultrafiltration liquid chromatography combined with counter-current chromatography. Talanta 2015, 134, 665-673. [CrossRef] [PubMed]

24. Zou, K.; Zhu, S.; Meselhy, M.R.; Tohda, C.; Cai, S.; Komatsu, K. Dammarane-type triterpene saponins from Panax japonicus. J. Nat. Prod. 2002, 65, 1288-1292. [CrossRef] [PubMed]

25. Nguyen, M.D.; Kasai, R.; Ohtani, K.; Ito, A.; Nguyen, T.N.; Yamasaki, K.; Tanaka, O. Saponins from Vietnamese Ginseng, Panax vietnamensis HA et Grushv. Collected in central Vietnam. II. Chem. Pharm. Bull. 1994, 42, 115-122. [PubMed]

26. Tanaka, O.; Han, E.C.; Yamaguchi, H.; Matsuura, H.; Murakami, T.; Taniyama, T.; Yoshikawa, M. Saponins of plants of Panax species collected in Central Nepal, and their chemotaxonomical significance. III. Chem. Pharm. Bull. 2000, 48, 889-892. [CrossRef] [PubMed]

27. Wan, J.B.; Zhang, Q.W.; Hong, S.J.; Li, P.; Li, S.P.; Wang, Y.T. Chemical investigation of saponins in different parts of Panax notoginseng by pressurized liquid extraction and liquid chromatography-electrospray ionization-tandem mass spectrometry. Molecules 2012, 17, 5836-5853. [CrossRef] [PubMed]

28. Zhang, Y.; Han, L.F.; Sakah, K.J.; Wu, Z.Z.; Liu, L.L.; Agyemang, K.; Gao, X.M.; Wang, T. Bioactive protopanaxatriol type saponins isolated from the roots of Panax notoginseng (Burk.) F. H. Chen. Molecules 2013, 18, 10352-10366. [CrossRef] [PubMed]

29. Xing, R.; Zhou, L.; Xie, L.; Hao, K.; Rao, T.; Wang, Q.; Ye, W.; Fu, H.; Wang, X.; Wang, G.; Liang, Y. Development of a systematic approach to rapid classification and identification of notoginsenosides and metabolites in rat feces based on liquid chromatography coupled triple time-of-flight mass spectrometry. Anal. Chim. Acta 2015, 867, 56-66. [CrossRef] [PubMed]

30. Liu, Y.; Li, J.; He, J.; Abliz, Z.; Qu, J.; Yu, S.; Ma, S.; Liu, J.; Du, D. Identification of new trace triterpenoid saponins from the roots of Panax notoginseng by high-performance liquid chromatography coupled with electrospray ionization tandem mass spectrometry. Rapid Commun. Mass Spectrom. 2009, 23, 667-679. [CrossRef] [PubMed]

31. Danieli, B.; Falcone, L.; Monti, D.; Riva, S.; Gebhardt, S.; Schubert-Zsilavecz, M. Regioselective enzymatic glycosylation of natural polyhydroxylated compounds: Galactosylation and glucosylation of protopanaxatriol ginsenosides. J. Org. Chem. 2001, 66, 262-269. [CrossRef] [PubMed]

32. Wang, H.P.; Yang, X.B.; Yang, X.X.; Liu, J.X.; Wang, Y.P.; Zhang, L.X. Chemical constituents from roots and rhizomes of Panax ginseng cultivated in Jilin province. China J. Chin. Mater. Med. 2013, 38, 2807-2817. 
33. Sun, S.; Qi, L.W.; Du, G.J.; Mehendale, S.R.; Wang, C.Z.; Yuan, C.S. Red notoginseng: Higher ginsenoside content and stronger anticancer potential than Asian and American ginseng. Food Chem. 2011, 125, 1299-1305.

34. Wang, J.R.; Yau, L.F.; Zhang, R.; Xia, Y.; Ma, J.; Ho, H.M.; Hu, P.; Hu, M.; Liu, L.; Jiang, Z.H. Transformation of Ginsenosides from Notoginseng by Artificial Gastric Juice Can Increase Cytotoxicity toward Cancer Cells. J. Agric. Food Chem. 2014, 62, 2558-25573. [CrossRef] [PubMed]

35. Kim, J.H.; Han, Y.N. Dammarane-type saponins from Gynostemma pentaphyllum. Phytochemistry 2011, 72, 1453-1459. [CrossRef] [PubMed]

36. Qiu, F.; Ma, Z.Z.; Xu, S.; Yao, X.S.; Chen, Y.J.; Che, Z.T. Studies on dammarane-type saponins in the flower-buds of Panax ginseng C.A. Meyer. J. Asian Nat. Prod. Res. 1998, 1, 119-123. [CrossRef] [PubMed]

37. Kouichi, Y.; Hari, P.D.; Hiroharu, F.; Shoji, Y. Saponins Composition of Rhizomes, Taproots, and Lateral Roots of Satsuma-ninjin (Panax japonicus). Chem. Pharm. Bull. 2013, 61, 344-350.

38. Wang, J.R.; Yau, L.F.; Tong, T.T.; Feng, Q.T.; Bai, L.P.; Ma, J.; Hu, M.; Liu, L.; Jiang, Z.H. Characterization of Oxygenated Metabolites of Ginsenoside Rb1 in Plasma and Urine of Rat. J. Agric. Food Chem. 2015, 63, 2689-2700. [CrossRef] [PubMed]

39. Ruan, C.C.; Liu, Z.; Li, X.; Liu, X.; Wang, L.J.; Pan, H.U.; Zheng, Y.N.; Sun, G.Z.; Zhang, Y.S.; Zhang, L.X. Isolation and Characterization of a New Ginsenoside from the Fresh Root of Panax ginseng. Molecules 2010, 15, 2319-2325. [CrossRef] [PubMed]

40. Xu, F.X.; Yuan, C.; Wan, J.B.; Yan, R.; Hu, H.; Li, S.P.; Zhang, Q.W. A novel strategy for rapid quantification of 20(S)-protopanaxatriol and 20(S)-protopanaxadiol saponins in Panax notoginseng, P. ginseng and P. quinquefolium. Nat. Prod. Res. 2015, 29, 46-52. [CrossRef] [PubMed]

41. Chen, B.; Wei, Y.J.; Wang, D.D.; Jia, X.B. Metabolism of ginsenosides Rk3 and Rh4 from steamed notoginseng in zebrafish by ultraperformance liquid chromatography/quadrupole-time-of-flight mass spectrometry. Arch. Pharm. Res. 2015, 38, 1468-1476. [CrossRef] [PubMed]

42. Patel, D.N.; Lin, H.S.; Koh, H.L. Quantification of ginsenosides Rh4 and Rk3 in rat plasma by liquid chromatography-tandem mass spectrometry: Application to a pre-clinical pharmacokinetic study. J. Mass. Spectrom. 2012, 47, 1510-1517. [CrossRef] [PubMed]

43. Nguyen, M.D.; Nguyen, T.N.; Kasai, R.; Ito, A.; Yamasaki, K.; Tanaka, O. Saponins from Vietnamese ginseng, Panax vietnamensis Ha et Grushv. Collected in central Vietnam. I. Chem. Pharm. Bull. 1993, 41, 2010-2014. [PubMed]

44. Han, L.F.; Sakah, K.J.; Liu, L.; Kojo, A.; Wang, T.; Zhang, Y. Saponins from Roots of Panax notoginseng. Chin. Herb. Med. 2014, 6, 159-163. [CrossRef]

Sample Availability: Samples of the compounds are available from the authors.

(C) 2015 by the authors; licensee MDPI, Basel, Switzerland. This article is an open access article distributed under the terms and conditions of the Creative Commons by Attribution (CC-BY) license (http://creativecommons.org/licenses/by/4.0/). 